\title{
Age-related inhibitory deficit, or lack of familiarity benefit? Evidence from letter identification among visual distractors
}

\author{
Cristina D. Rabaglia $^{1}$ (D) Bruce A. Schneider ${ }^{1}$
}

Published online: 5 November 2015

(C) The Psychonomic Society, Inc. 2015

\begin{abstract}
Age-related deficits in processing complex visual scenes are often attributed to age-related declines in the cognitive abilities required for such tasks. For example, poorer or slower performance of a complex task in the presence of distractor items is often attributed to an age-related deficit in the ability to inhibit the processing of irrelevant information. To investigate the relative contributions of sensory and cognitive factors in such tasks, younger and older participants were asked to identify a letter presented simultaneously with distractors that were either other letters, pieces of letters, or visual noise controls with identical spatial frequency content or contrast profile. In Experiment 1, older adults performed much worse than younger adults when the masking field consisted of other letters. Surprisingly - and contrary to the predictions of inhibitory deficit or visual "pop-out" phenomena-this effect emerged because younger adults performed much better with letter-containing maskers than with any other type of masker, whereas older adults did not. Experiment 2 revealed that age-related changes in the time required to process the visual display do not appear to account for this effect. In Experiment 3, however, we replicated older adults' task performance in a younger adult sample by filtering the experimental stimuli to match the image contrast typically experienced by an older adult. The results of Experiment 3 suggest that age-related differences in task performance amidst distractors can emerge from age-related declines in contrast sensitivity, which set older adults up to fail at tasks in which
\end{abstract}

Cristina D. Rabaglia

cris.rabaglia@utoronto.ca

1 Department of Psychology, University of Toronto Mississauga, Mississauga, Ontario, Canada younger adults may typically be able to benefit from the familiarity of the target and surrounding objects.

Keywords Aging · Inhibitory deficit · Visual object formation · Sensory-cognitive interactions · Letter identification

One of the goals of cognitive aging research is to identify the reasons for the poorer performance of older adults on a number of laboratory-administered tests of diverse cognitive functions such as memory, reasoning, and language comprehension. Many of these age-related declines are often attributed to age-related deficits in inhibiting the processing of irrelevant information (e.g., Hasher \& Zacks, 1988; Lustig, Hasher, \& Zacks, 2007).

A majority of older adults, for example, report difficulty comprehending conversations when multiple people are talking (Pichora-Fuller, 1997); under an inhibitory deficit account, this difficulty would stem from an age-related decline in the ability to ignore or inhibit co-occurring, but irrelevant, speech while engaged in a conversation. If this irrelevant speech gains access to working memory, it could compete with the relevant, target speech for attentional resources. Therefore an age-related lessening of the ability to inhibit the entry and/or processing of irrelevant material in working memory will lead to performance declines in older adults with respect to the primary task of comprehending the conversation. Since the completion of almost any cognitive task involves the balancing of multiple potential sources of information, such inhibitory deficits could have widespread effects across many aspects of cognitive life.

The first experimental tests of this hypothesis - and the majority of experimental support for it since-have come from studies involving "reading with distraction," which 
require participants to read selections of text presented simultaneously with irrelevant material (Carlson, Hasher, Connelly, \& Zacks, 1995; Connelly, Hasher, \& Zacks, 1991; Duchek, Balota, \& Thessing, 1998; Mund, Bell, \& Buchner, 2010, 2012). Consistent with the inhibitory deficit hypothesis, such studies usually show that older adults are more detrimentally affected by the presence of distracting text, particularly if the distracting text is semantically related to the target text. For older adults, the presence of such distractors results in greater reductions in reading speed (Carlson et al., 1995; Connelly et al., 1991) and/or text comprehension (Mund et al., 2010), relative to younger adults.

In the typical experimental setup for such reading-withdistraction studies, the distracting text is interspersed within the target text, with distracting text being differentiated from the target text through the use of different font types or emphasis (e.g., italicizing one but not the other). The interpretability of the experimental effects as reflecting different inhibitory abilities, therefore, is contingent on older and younger adults being equally able to distinguish the two types of fonts or emphasis. However, a wide variety of changes that occur in the aging visual system virtually ensure that the visual inputs available to higher-order processing during reading of text are not equivalent between older and younger adults. Older adults, including those whose vision is clinically normal, typically experience decreased retinal illuminance due to pupillary miosis and increased crystalline lens density, increases in optical aberrations and intra-ocular light scatter that can reduce image contrast, and reductions in contrast sensitivity, particularly for high-spatial-frequency images (see Owsley, 2011). Under dim lighting conditions, for example, older adults need three times the contrast to achieve levels of performance equivalent to those of younger adults in detecting grating orientations (Schefrin, Tregear, Harvey, \& Werner, 1999), and require nearly twice the font size needed by younger adults to read without impediment when contrast levels are low (Steenbekkers, 1998).

Precisely how different the incoming visual inputs may be for older and younger adults in experiments employing visual stimuli usually remains unknown, because even simple measures, such as acuity and contrast sensitivity, are rarely collected and reported for older and younger adult age groups (Schneider \& Pichora-Fuller, 2000). If a portion of the apparent inhibitory deficit exhibited by older adults were in fact due to age-related declines in vision, we would expect that any manipulation that increased the distinctiveness between the target text and the interfering text should reduce the magnitude of the apparent inhibitory deficit. Indeed, manipulations that decrease the necessity to differentiate between italicized and nonitalicized fonts, by presenting the distracting text in a distinct font color (Kemper, McDowd, \& Kramer, 2006; Phillips \& Lesperance, 2003) or in a consistent location spatially distinct from that of the target text (Carlson et al., 1995), eliminate age differences in the effect of distractors.

Another factor that could be in play in the reading-withdistraction paradigm is the well-known visual phenomenon of pop-out. Pop-out refers to the fact that, in a visual display, items that have distinguishing visual features - such as color or shape-tend to "pop out" from the rest of the items in the display; as a result, these items are easy to identify and tend to take very little time to find (e.g., Treisman \& Gelade, 1980). In typical experimental paradigms used to investigate the inhibitory deficit hypothesis, the lower-level visual features of the targets and distractors (e.g., orientation, in slanted vs. no slant for italicized letters) are often purposefully manipulated, as the mechanism through which the target is differentiated from the distracting text. To the extent that the visual systems of younger adults provide robust representations of the lower-level features that may distinguish the target from the distracting font, the more likely it is that the target text will be effortlessly distinguished (pop out) from the background provided by the distracting text. If older adults have comparatively impoverished or degraded incoming visual features-because of age-related visual declines and loss of contrast sensitivity - they may not experience a pop-out effect in such experimental scenarios. As a result, older adults may not be able to effortlessly and automatically distinguish targets from distractors as younger adults can-not due to an inhibitory issue, per se, but rather because of lower-order visual factors. Hence, although reading-withdistraction task results are consistent with the emergence of age-related inhibitory deficits, the degree to which agerelated changes in lower-order visual functioning are responsible for the age-related deficits in these reading tasks remains unclear.

Interpretational difficulties are also present in readingwith-distraction tasks due to the nature of the paradigm itself. When participants are charged with reading entire passages of text in the presence of visual distraction, age-related differences in performance, in addition to reflecting age-related differences in inhibition and/or in visual processing, could also be influenced by age-related differences in higher-level strategies. For example, if older adults are more likely or better able than are younger adults to use top-down strategies to retain comprehension in the face of processing difficulties, they may exhibit fewer age differences in comprehension performance, even though the reading of the text with distraction is, in fact, more disruptive. On the other hand, if older adults are more likely to weigh accuracy over speed-as some work suggests (Brebion, 2001, 2003) - then older adults may show slowed reading times under distracting conditions because of related shifts in the speed-accuracy criterion, rather than specific difficulty with inhibiting the content of the co-occurring text. 
An alternative to the typical reading-with-distraction of passage-level text that is less susceptible to these potential confounds, then, is a simple letter identification task. In this task, a single letter in isolation is presented in the middle of a visual display, and participants must respond with the identity of the displayed letter. The visual display can also consist of a variety of types of simultaneously displayed maskers (Fig. 1). In this task, the target letters are not embedded in words, and thus stimulus identification does not require the engagement of the myriad higher-order semantic and linguistic processes that are required by reading text. Additionally, by varying the signal strength of the to-be-identified target letter, we can assess the degree to which the psychometric functions relating identification to signal strength differ with age. In particular, we can use these functions to determine the signal strength required to reach some specified level of performance and compare these values across age groups, thereby eliminating the need to rely on age-related processing time differences that are potentially confounded by age-related differences in speed-accuracy criterion. The slopes of these psychometric functions can also provide insight into the potential degree of perceptual confusion between the target letters and different types of co-occurring visual distraction, since we would expect that the more distinct the masker is from the target, the steeper the slope of the psychometric function would be.

In addition to being less susceptible to the potential confounds found in reading-with-distraction tasks, the letter identification paradigm should be quite informative as to the sensory factors likely to limit theoretically higher-order tasks such as reading. For example, Pelli and Tillman (2007) simulated within individuals the consequences of misidentifying letters, by substituting similar letters for the correct letters (e.g., presenting the word "hewane" instead of "beware" in a passage), and found that this type of substitution had a profound effect on reading speed. Hence, we would expect that age-related changes in vision that lead to letter confusion would limit reading speed and comprehension in older adults. Chung and Tjan (2009) have also shown that the relationship between the factors limiting letter identification and reading are especially tightly linked when contrast levels are

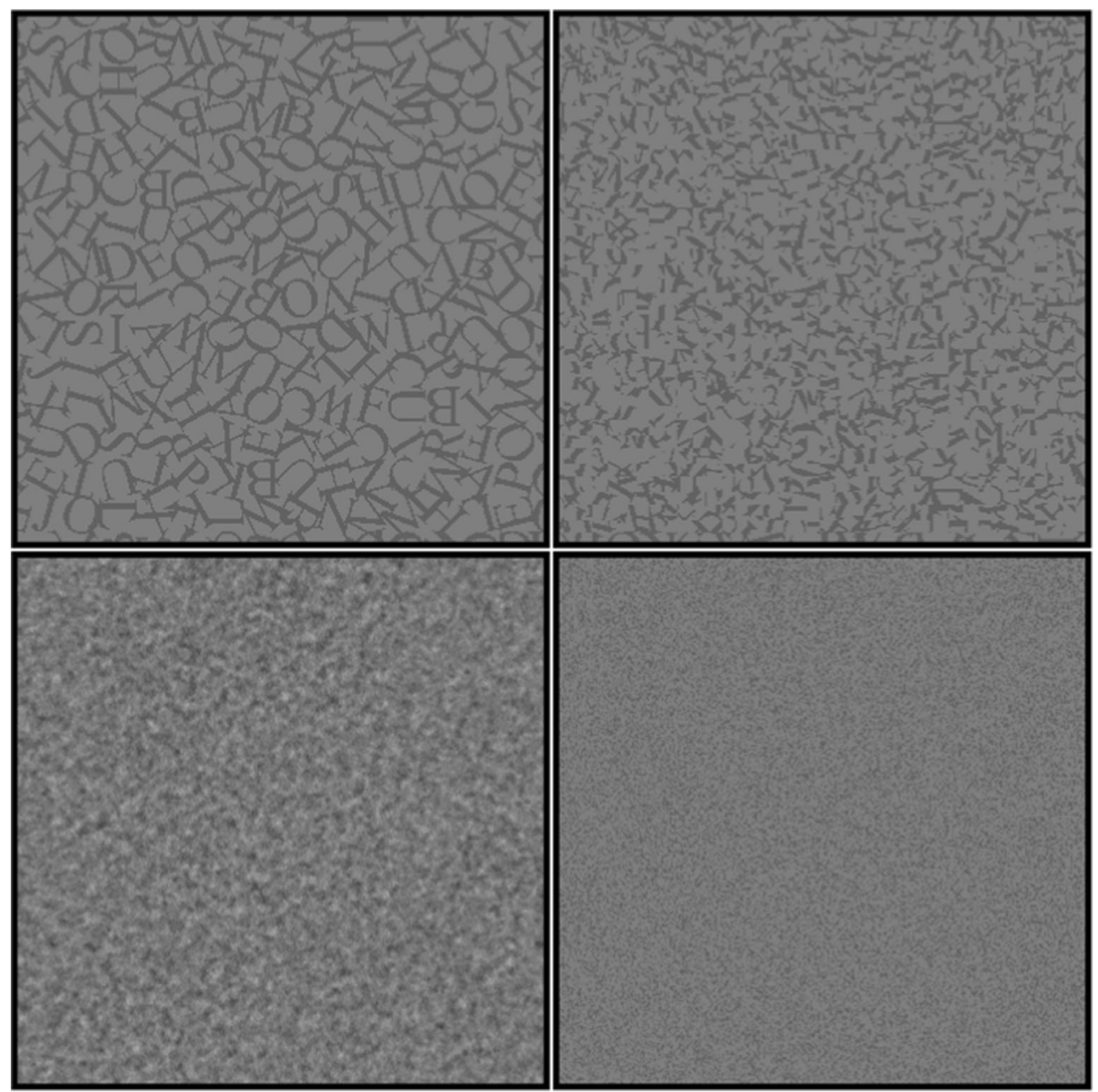

Fig. 1 Examples of the four maskers used for all experiments. The maskers are, in clockwise order from top left, the letter masker, fragment masker, pixel masker, and phase masker 
low. Because of age-related changes in visual processing, the effective level of contrast in a display is likely to be lower for older than for younger adults, leading to poorer discriminability between similarly shaped letters (e.g., b and h).

In the experiments reported here, we investigated the impact of co-occurring information on task performance in older and younger adults in a letter identification task. In this task, participants were asked to identify a target letter that was simultaneously presented with one of four different types of visual distractors (maskers) that varied in their degrees of spatial organization and relationship to the target letter: a letter masker, composed of randomly oriented letters; a fragment masker, composed of randomly oriented pieces of letters; a phase masker, composed of visual noise with identical spatial frequency content to that of the letter masker, but without discernable contours or objects; and a pixel masker, created by randomly permuting the pixel locations in the letter masker.

If older adults do experience more interference from meaningful distracting material, we would expect that age-related differences in performance would be larger in the letter masker condition, relative to the perceptually similar but semantically meaningless fragment and phase masker conditions. For both older and younger adults, we also expected - consistent with findings in the pop-out literature-performance to be worst in the letter masker condition, in which the target was most similar, perceptually and semantically, to the concurrently presented masker. We were therefore quite surprised when the results turned out to be almost precisely the opposite, with respect to the latter prediction.

\section{Experiment 1}

\section{Method}

Participants Twelve younger adults ranging in age from 18 to 25 years old $(M=20.2$ years, $S D=2.4)$ and 12 older adults ranging from 65 to 76 years old $(M=70.0$ years, $S D=4.0)$ completed the study in exchange for a modest honorarium. All participants were self-identified native speakers of English who indicated, via self-report on a questionnaire, no history of neurological or ocular pathology.

All participants completed an in-lab vision screening (Table 1), including near and far acuity and contrast sensitivity, via LogMAR acuity [LogMAR = $-\log$ (decimal acuity)] and F.A.C.T. contrast sensitivity slides presented using an Optec 6500P (Stereo Optical Company, Chicago, IL). During the vision screening, participants wore their own appropriate corrective lenses when necessary. All participants had near acuity of 20/ 25 (decimal acuity $=.8$ ) or better, but the younger adult group did have significantly better near acuity, $t(22)=$ 2.66, $p=.018$; far acuity in the two groups did not differ significantly, $t(22)=0.65, p=.524$. Note that to take into account the logarithmic nature of the acuity scale, all descriptive statistics and acuity-related significance tests were computed on LogMAR values. Older adults had significantly worse overall contrast sensitivity performance (Table 1) than did younger adults, $t(22)=$ $3.09, p=.005$ (average contrast sensitivity collapsed across spatial frequencies).

Table 1 Visual acuity and contrast sensitivity descriptive statistics for the older and younger adult samples in all experiments

\begin{tabular}{|c|c|c|c|c|c|c|c|}
\hline & \multicolumn{2}{|l|}{ Acuity } & \multicolumn{5}{|c|}{ Contrast Sensitivity } \\
\hline & Near & Far & 1.5 & 3 & 6 & 12 & 18 \\
\hline \multicolumn{8}{|l|}{ Experiment 1} \\
\hline YA & $1.40 \pm 0.94$ & $1.41 \pm 0.76$ & $.95(.10)$ & $.91(.13)$ & $.96(.05)$ & $.92(.11)$ & $.88(.20)$ \\
\hline $\mathrm{OA}$ & $1.23 \pm 1.14$ & $1.19 \pm 1.30$ & $.87(.11)$ & $.84(.12)$ & $.86(.17)$ & $.66(.25)$ & $.59(.27)$ \\
\hline \multicolumn{8}{|l|}{ Experiment $1 \mathrm{~b}$} \\
\hline YA & $1.50 \pm 0.50$ & $1.19 \pm 0.88$ & $.92(.08)$ & $.88(.15)$ & $.86(.18)$ & $.88(.13)$ & $.71(.34$ \\
\hline $\mathrm{OA}$ & $1.10 \pm 0.97$ & $1.00 \pm 1.30$ & $.89(.12)$ & $.81(.08)$ & $.83(.10)$ & $.61(.12)$ & $.46(.07)$ \\
\hline \multicolumn{8}{|l|}{ Experiment 2} \\
\hline YA & $1.51 \pm 0.36$ & $1.36 \pm 0.78$ & $.97(.05)$ & $.93(.11)$ & $.96(.10)$ & $.91(.12)$ & $.87(.23$ \\
\hline $\mathrm{OA}$ & $1.02 \pm 0.81$ & $1.11 \pm 0.91$ & $.89(.09)$ & $.81(.13)$ & $.81(.19)$ & $.70(.20)$ & $.53(.24$ \\
\hline \multicolumn{8}{|l|}{ Experiment $3^{\text {a }}$} \\
\hline YA & $1.22 \pm 0.99$ & $1.12 \pm 1.42$ & $.90(.12)$ & $.87(.15)$ & $.88(.14)$ & $.75(.27)$ & $.68(.33$ \\
\hline OA $250 \mathrm{~ms}$ & $1.22 \pm 0.99$ & $1.22 \pm 0.99$ & $.90(.11)$ & $.82(.14)$ & $.83(.21)$ & $.74(.22)$ & $.51(.28$ \\
\hline OA $1 \mathrm{~s}$ & $1.22 \pm 0.99$ & $1.15 \pm 1.20$ & $.86(.10)$ & $.86(.10)$ & $.86(.17)$ & $.64(.28)$ & $.58(.28$ \\
\hline
\end{tabular}

$\mathrm{YA}=$ younger adult; $\mathrm{OA}=$ older adult. Means are presented with standard deviations in parentheses. Acuity is presented as the mean decimal acuity \pm standard deviation in lines of LogMAR acuity, calculated as discussed in the main text. Contrast sensitivity is presented as the percentage of correctly identified orientations of gratings, separately for each of the five tested spatial frequencies in cycles per degree (cpd). ${ }^{\text {a }}$ The descriptives for older adults are for the comparison subsets drawn from Experiment $1(1,000 \mathrm{~ms})$ and Experiment $2(250 \mathrm{~ms})$ 
Materials Participants saw a visual field subtending $12.26 \times$ $12.26 \mathrm{deg}$ of visual angle with a mid-gray background luminance (luminance set to the middle of the possible pixel luminance range). Target letters were presented in the center of this field. All target letters appeared as Times New Roman capital letters. The visual angle subtended by the heights of all but one of these capital letters (the letter Q) was $0.86 \mathrm{deg}$. The height of the visual angle subtended by the letter Q was $1.1 \mathrm{deg}$. The widths of the visual angles of the letters ranged from $0.28 \mathrm{deg}$ (the letter I) to $1.1 \mathrm{deg}$ (the letter W). The target and masking letters were displayed in a modified version of Times New Roman font, such that when the targets were scanned for inclusion into the display, if a border fell across the edges of a single pixel, the luminance value for that pixel was classified according to the value of either the background field or the letter, depending on what portion of the pixel was occupied by that portion of the display: If over $50 \%$ of the pixel was included in the letter, the entire pixel received the luminance value for the letter; if over $50 \%$ of the pixel was included in the background field, the entire pixel received the luminance value for the background. This ensured that the contrast value for the entire letter was uniform and entirely conformed to the specified pixel luminance of that letter.

Target letters were displayed simultaneously with one of four possible masker types (Fig. 1). For these experiments, 100 maskers of each type were created using a custom Mathematica script. Letter maskers (described below) were created first; individual maskers for all of the other masker types were formed as transformations over each individual letter masker.

In the letter masker condition, the masker was composed of randomly selected, randomly oriented letters, each with a Weber contrast of $C=.2117$ (see below) from the background mid-gray of the visual field. To populate the spatial area of the masker with letters, the visual field was divided into a $15 \times 15$ grid, and the spatial location, orientation, and identity of the letters within each cell of the grid were randomly selected for each masker, subject to the restriction that no letter in the masker could appear in the canonical (vertical) orientation.

In the pixel masker condition, the root mean square (RMS) pixel contrast ${ }^{1}$ of each letter masker was computed, and the individual pixel luminance values in the letter masker were randomly distributed over the entire visual field, creating a masker with the same overall RMS contrast as the letter masker.

Maskers for the phase masker condition were created by taking the 2-D Fourier transform of the letter masker image and computing the amplitude and phase components for each spatial frequency. The phase value for each spatial frequency was replaced with a random value between $-\pi$ and $\pi$. These

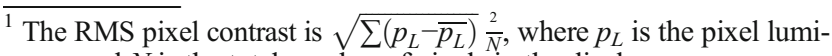
nance and $N$ is the total number of pixels in the display.
}

original amplitude and randomized phase components were then recombined to create a reconstituted masker image, thus retaining the overall RMS contrast and spatial-frequency components of the original image, but with randomized phase. This transformation creates an image with equivalent spatial frequency and overall contrast, but without recognizable objects (because the phase component-corresponding to the relative position of the individual spatial-frequency components within the image - has been randomized).

In the fragment masker condition, each letter in the letter masker was transected into four segments, which were then randomly rotated and randomly placed at a spatial position within its original grid cell, with the exception that this random rotation and spatial displacement could not re-form it into the same original letter.

Figure 2 presents the averages of the amplitude spectra for the 100 maskers. Note that the letter masker and the random phase masker have the same average amplitude spectra. The fragment masker has energy in the same frequency region as that of the letter masker and phase masker, but its average amplitude spectrum is not precisely the same as the latter two. Finally, because the pixels were randomly relocated in the pixel masker, the spectrum of the pixel masker is flat. Note, however, that all images have the same RMS pixel contrast.

Procedure Participants sat in a dimly lit soundproof booth in front of a Dell Inspiron 660 desktop computer and a 16-in. SONY GDM-F250 screen with an $85-\mathrm{Hz}$ refresh rate. Participants' head position was stabilized with a chinrest, placed 16 in. away from the computer screen.

A trial in each condition began with a square fixation point subtending $0.07 \times 0.07 \mathrm{deg}$ of visual angle and lasting for $500 \mathrm{~ms}$, followed by the target letter and the masker simultaneously for $1,000 \mathrm{~ms}$, and then by a 500 -ms backward mask consisting of random dots composed of the two extreme pixel luminance (maximum and minimum) values. Participants were instructed to identify the letter presented by typing in their selection using a keyboard; responses were displayed on the screen via a textbox that appeared only after the letter and masker had disappeared, and participants pressed the return key to $\log$ their answer. Participants were informed in a feedback box (which lasted for approximately $1 \mathrm{~s}$ ) whether their response was correct or wrong. The next trial sequence began immediately after the feedback box had disappeared.

Target letters were presented at one of four equally spaced contrast levels in each condition (see Table 2 for the contrast levels for all experiments). The four contrast levels used for each condition, in each age group, were chosen on the basis of pilot participants in each age group, such that we expected the percentage of correct identifications to be less than $50 \%$ for the two lowest contrast values, and higher than $50 \%$ for the two highest values. We then used these contrast values to 

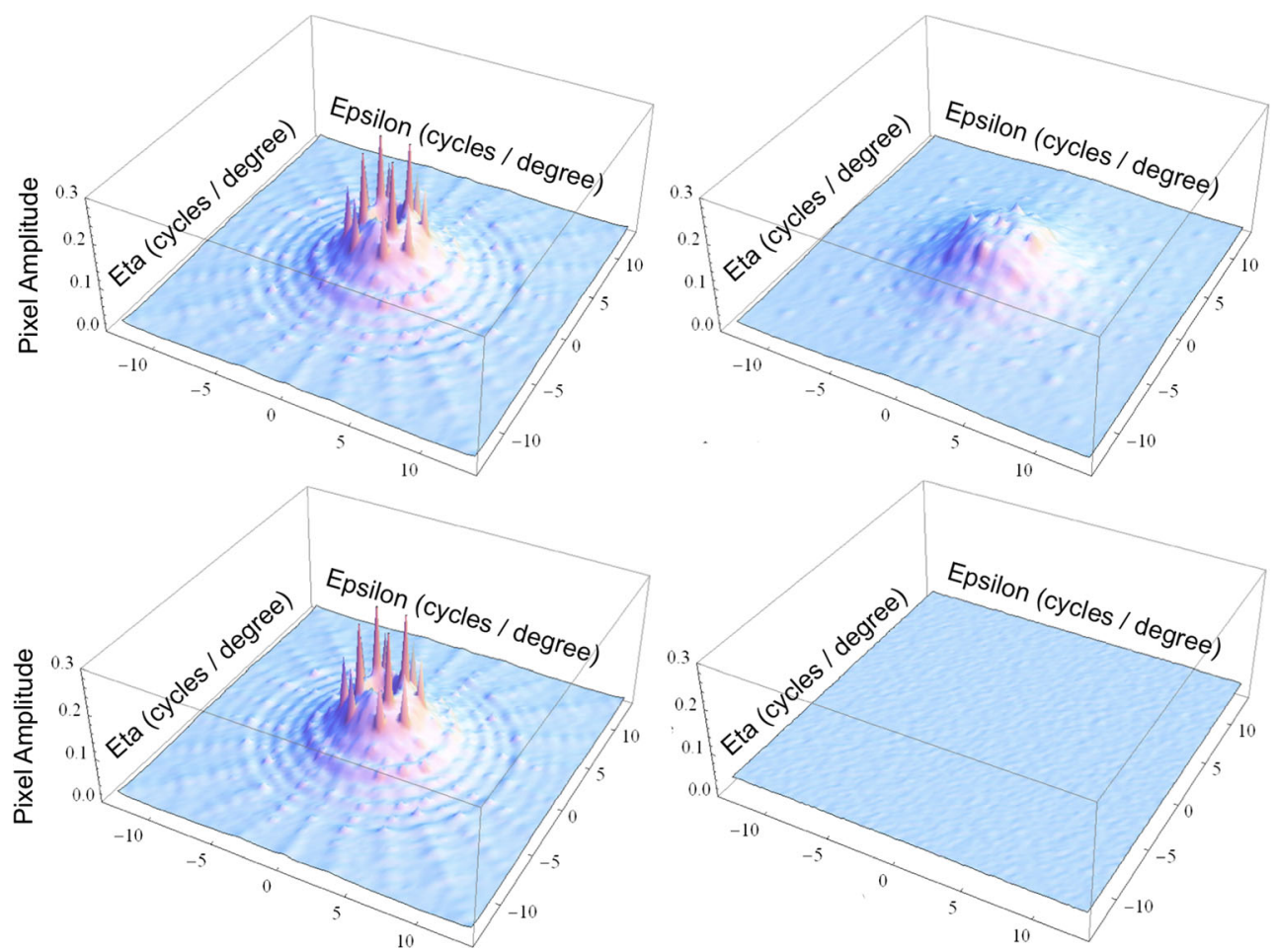

Fig. 2 Average spectra profiles across all 100 maskers used for each of the four masker conditions in Experiments 1 and 2. The maskers are, in clockwise order from top left, the letter masker, fragment masker, pixel masker, and phase masker

Table 2 The four Weber contrast levels used for each masker condition and age group in Experiments 1, 2, and 3

\begin{tabular}{lll}
\hline & Younger Adults & Older Adults \\
\hline Experiment 1 & & \\
$\quad$ Letter & $.016, .031, .047, .063$ & $.024, .118, .213, .307$ \\
Fragment & $.024, .055, .087, .118$ & $.094, .165, .236, .307$ \\
Phase & $.079, .118, .157, .197$ & $.102, .157, .213, .268$ \\
$\quad$ Pixel & $.039, .071, .102, .134$ & $.039, .079, .119, .159$ \\
Experiment 2 & & \\
Letter & $.008, .031, .055, .079$ & $.024, .173, .323, .472$ \\
Fragment & $.016, .063, .110, .157$ & $.024, .173, .323, .472$ \\
Phase & $.055, .110, .165, .220$ & $.039, .173, .307, .441$ \\
$\quad$ Pixel & $.031, .079, .126, .173$ & $.039, .118, .197, .276$ \\
Experiment 3 & & \\
$\quad$ Letter & $.024, .173, .323, .472$ & - \\
Fragment & $.024, .173, .323, .472$ & - \\
Phase & $.039, .173, .307, .441$ & - \\
Pixel & $.024, .094, .165, .236$ & - \\
\hline
\end{tabular}

Contrast values were input into the experimental program as evenly spaced whole numbers between 0 and 255, corresponding to reverse pixel luminance. The values presented here, the Weber contrast values for target letters, were calculated as described in the main text and rounded to three decimal places. For Experiment 1, the values used in the phase masker condition are valid both for the phase-transformed letter maskers (main experiment) and the follow-up with phase-transformed letter fragment maskers estimate the expected degree of variance in threshold measures and to determine, for a particular sample size, the between-subjects difference that would produce a power of .8. On the basis of these data, we selected a sample size of 12 (see the Appendix for further information). Target letter contrast was operationalized in terms of pixel luminance; contrast values were input into the custom Mathematica script as a pixel luminance integer value ranging from 0 (maximum luminance) to 255 (no luminance: black). For all analyses and results, the contrast, $C$, of the target letters was defined as the Weber contrast of the target letter relative to the mid-gray background, computed as $\left(L-L_{b}\right) / L_{b}$, where $L$ is the darkness of the target letter in terms of possible pixel luminance $(0$ being total possible illumination for a pixel, numbers higher than zero representing less pixel luminance) and $L_{b}$ is the darkness in terms of pixel luminance of the background. This created a representation of the contrast level of the target letter relative to the background that was bounded between $0(L=$ $\left.L_{b}\right)$ and $1\left(L=2 L_{b}\right)$, where values closer to 1 represented letters darker than the background.

Trials in each condition were blocked together. Participants completed two consecutive blocks of 100 trials in each of the four masker conditions. Each block of 100 trials contained, in a random order, 25 trials per each of four possible contrasts for the target letter; the target letter identity and individual maskers for each trial were selected randomly. Prior to the two experimental blocks in each condition, participants completed eight practice trials in a randomly presented order, two 
at each of the four possible contrasts for the condition. The order of the presentation of the four conditions was counterbalanced across the participants in each age group, such that each condition occurred at each relative order an equal number of times within each age group, and the possible transitions between conditions (e.g., letter $\rightarrow$ fragment; fragment $\rightarrow$ pixel, etc.) occurred an equal number of times. Each age group completed the same set of counterbalances.

\section{Results}

Figure 3 plots the proportions of correctly identified target letters, averaged across participants, as a function of the Weber contrast of the target letters, for the letter masker (top left panel), fragment masker (top right panel), phase masker (bottom left panel), and pixel masker (bottom right panel) conditions separately for younger (black circles) and older (gray triangles) adults. The smooth curves drawn through the points are the best-fitting logistic functions of the form

$y=\frac{1}{1+e^{-\sigma(x-\mu)}}$,

where $y$ is the probability of correctly identifying the target letter at a given contrast level $x, \mu$ is the contrast level corresponding to $50 \%$ correct target letter identification performance, and $\sigma$ governs the slope of the psychometric function.

The rightward horizontal shift along the $x$-axis (Fig. 3) of the older adults' functions (gray lines), relative to the younger adults' (black lines) indicates that older adults required higher contrast levels to achieve performance equivalent to that of younger adults in every masking condition. However, the

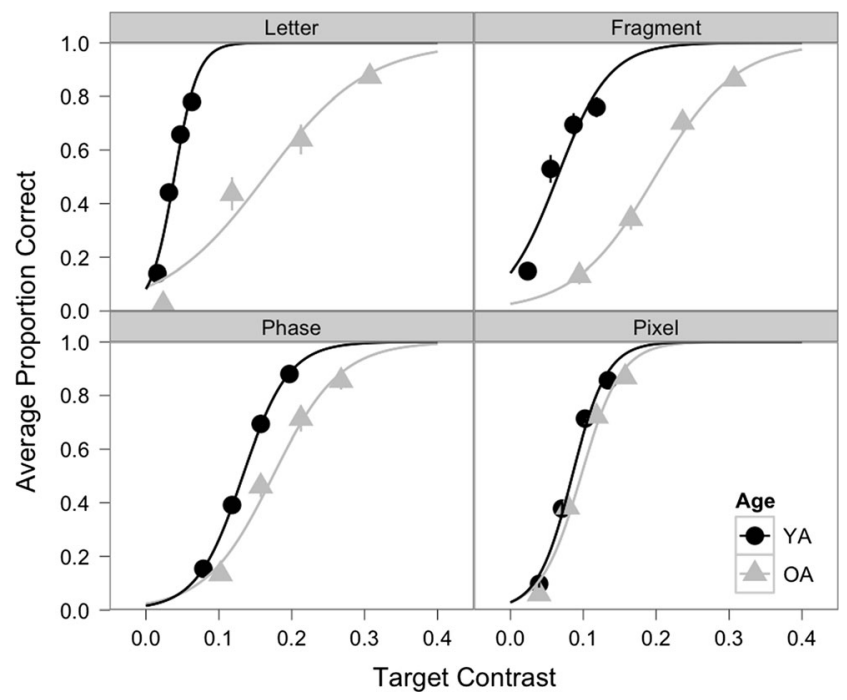

Fig. 3 Average proportion correct target letter identification as a function of contrast for younger and older adult age groups, in the four masker conditions. The data are displayed with psychometric functions fitted to the participant-average data in Experiment 1. Error bars represent \pm 1 $S E M$. YA = younger adult, $\mathrm{OA}=$ older adult horizontal distance between the two age groups' functions differed substantially between the different masking conditions, indicating that, as predicted, the effect of age on letter detection performance differed to some degree between the masker types.

In the pixel masker condition (bottom right panel, Fig. 3), the average fitted function to the older adults' data largely mirrors that of younger adults' in slope and is shifted only a small amount to the right. Recall that the pixel masker retained the RMS contrast of the letter masker, but randomized the locations of the pixels of the letter masker, creating a masking field of random noise with a flat spectrum. Hence, when the target letter was masked by flat-spectrum random noise, older adults needed only slightly higher-contrast target letters to perform equivalently to younger adults. The gap between older and younger adults' psychometric functions widens to a slight degree in the phase masker condition (bottom left panel, Fig. 3), with the slope of the older adults' psychometric function being slightly shallower than that of the younger adults. Note that this masker had the same spatialfrequency composition as the letter masker, but, because its phase spectrum was randomized, had no organized or predictable contours. Dramatic differences between the age groups emerge, however, in the letter and fragment masker conditions (top panels, Fig. 3). Relative to younger adults' average fitted functions, older adults' functions in these two conditions are much shallower and are shifted substantially to the right, indicating that older adults required much higher contrast levels than younger adults to achieve equivalent letter identification performance. In the letter masker condition (top left panel, Fig. 3), in particular, the differences in slope and threshold indeed appear the largest, indicating that in this condition older adults required a much higher signal strength (elevated $50 \%$ thresholds) and experienced the greatest relative interference from the masker (shallower slopes), as compared to younger adults.

Figure 3 also suggests that - within a given age groupidentification of the target letter was disrupted to differing degrees by the concurrent presentation of the different types of visual distractors. Looking at the younger adults' fitted functions only, the letter and fragment conditions are associated with leftward-shifted functions relative to the pixel and phase conditions; this is - surprisingly-particularly pronounced in the letter masker condition, which shows a leftward-shifted threshold and extremely steepened slope relative to the other three conditions. That is, contrary to our expectations, younger adults appear to be the least affected by the presence of the letter masker. For older adults, however, this pattern does not appear. Instead, older adults' functions appear relatively similar in the letter, fragment, and phase conditions, with similarly positioned $50 \%$ thresholds and relatively shallow slopes. 
A series of mixed-design analyses of variance (ANOVAs) on the threshold and slope parameters for the psychometric functions, fitted to the individual participant data, ${ }^{2}$ confirm the patterns demonstrated in the average data plotted in Fig. 3. To equalize the variance across experimental conditions, all individual thresholds and slopes were log-transformed prior to analysis. The individual psychometric function thresholds, $\mu$, were analyzed using a mixed-design ANOVA with Age Group (young, old) as a between-subjects factor and Masker Type (letter, pixel, phase, fragment) as a within-subjects factor. This analysis demonstrated large main effects of both age group, $F(1,21)=174.72, p<.001, \eta_{\mathrm{p}}^{2}=.893$, and masker condition, $F(2.40,47.60)=96.77, p<.001, \eta_{\mathrm{p}}{ }^{2}=.822$, as well as a significant age by masker type interaction, $F(2.27,47.60)$ $=124.24, p<.001, \eta_{\mathrm{p}}{ }^{2}=.855$, indicating that the effects of masker condition differed for older and younger adults. Follow-up pair-wise comparisons between the thresholds for each age group in each of the four masker conditions (see Table 3 for descriptive statistics) revealed that older adults had significantly higher $50 \%$ thresholds than did younger adults for every masker type (all $p \mathrm{~s}<.01$ ), although the magnitude of the relationship between age and threshold varied dramatically between masker conditions (Fig. 4).

To specify more precisely the nature of the interactions between age and masker type for the psychometric function thresholds, we compared the relative difficulty of each masker condition for each age group separately. For each age group, one-way within-subjects ANOVAs revealed significant and large-magnitude effects of the masker (Table 3, final three columns), indicating that within each age group, $50 \%$ contrast thresholds varied significantly across the four masker types. Follow-up pair-wise comparisons of the maskers (Table 4) within each age group indicated that, for both age groups, the phase masker was associated with significantly higher thresholds than the pixel masker. However, for younger adults, the fragment and letter masker conditions had significantly lower thresholds than the phase and pixel conditions, with the letter masker requiring the lowest contrast levels out of all the masker conditions (all $p$ s $<.01$ ). In sharp contrast to this pattern, among the older adult age group - although the letter masker did have a significantly lower threshold than the fragment masker - both the letter and fragment maskers required significantly higher contrast levels than the pixel masker $(p s<.001)$, and did not significantly differ from the phase masker thresholds $(p \mathrm{~s}>.50)$.

The individual slope parameters, $\sigma$, were analyzed in a parallel approach, using a mixed-design ANOVA with Age Group (young, old) as a between-subjects factor and Masker

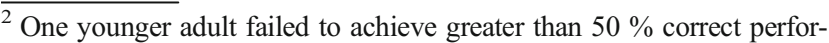
mance at the darkest contrast for the letter fragment condition; we thus could not fit a psychometric function to this participant's data in this condition. Data from this condition for this participant are not included in the analyses described below.
}

Condition (letter, pixel, phase, fragment) as a betweensubjects factor. This analysis revealed highly significant main effects for age group, $F(1,21)=196.92, p<.001, \eta_{\mathrm{p}}{ }^{2}=.904$, and masker condition, $F(3,63)=31.94, p<.001, \eta_{\mathrm{p}}^{2}=.603$, as well as a significant age by masker condition interaction, $F(3,63)=52.03, p<.001, \eta_{\mathrm{p}}{ }^{2}=.712$, indicating that the effects of masker condition on slopes differed between older and younger adults. Pair-wise comparisons of the younger and older adults' slopes in each of the four masker conditions (see Table 3 for descriptives) revealed that older adults' psychometric functions had significantly shallower slopes than the younger adults' in every masker condition (all $p$ s $<.05$ ) except the pixel condition, in which the difference between the older and younger adults' slopes ( $0.13 \mathrm{log}$ units) was only marginally significant, $t(22)=1.77, p=.091$. Reflecting the age by condition interaction, the magnitude of these age-related differences varied by condition (Fig. 3); the gap between older and younger adults' slopes widened to a slight degree successively across the pixel, phase, and fragment masker conditions, but increased dramatically in the letter masker condition. Within-subjects ANOVAs comparing the effects of masker type on slopes, conducted separately for each age group, revealed significant main effects of masker type for both age groups (Table 3, final three columns), but followup pair-wise comparisons of the slopes in the different masker conditions, performed within each of the two age groups separately, confirmed that the effect of masker condition diverged dramatically for older and younger adults in the letter masker condition. These pair-wise comparisons (see Table 5) revealed similar relative influences of masker condition on slopes for the pixel, fragment, and phase conditions for both age groups. In both younger and older adults, slopes were significantly steeper in the pixel masker condition than in the phase and fragment masker conditions, suggesting that masker-target segregation was more successful for both age groups with a flat-spectrum random-noise masker than with maskers with organized (fragment masker) and/or similar (phase masker) spatial frequency content. For younger adults only, however, the letter masker was associated with the steepest slope relative to other masking conditions (all $p \mathrm{~s}<.001$ ); for older adults, the letter masker had the shallowest slope, differing significantly from the pixel and phase, but not from the fragment, conditions ( $p=.153)$.

\section{Discussion}

The presence of maskers during a letter identification task, in the experiment described above, clearly had an effect on participants' ability to identify the letter. Collapsing across different specific types of maskers, the main effect of age found in this experiment on both psychometric function thresholds and slopes indicates that older adults were more susceptible to masking than were younger adults, and may have experienced 
Table 3 Descriptive statistics and results of follow-up within-subjects ANOVAs performed within each age group in Experiment 1

\begin{tabular}{|c|c|c|c|c|c|c|c|}
\hline & Letter & Fragment & Phase & Pixel & $d f$ & $F$ & $\eta_{\mathrm{p}}^{2}$ \\
\hline \multicolumn{8}{|l|}{ YA } \\
\hline$\mu$ & $.040(.007)$ & $.062(.015)$ & $.133(.015)$ & $.085(.006)$ & $(1.92,19.18)^{\mathrm{a}}$ & $153.67^{*}$ & .939 \\
\hline$\sigma$ & $63.554(7.611)$ & $31.184(5.584)$ & $32.502(4.496)$ & $42.341(6.901)$ & $(3,30)$ & $47.33^{*}$ & .826 \\
\hline \multicolumn{8}{|l|}{$\mathrm{OA}$} \\
\hline$\mu$ & $.165(.050)$ & $.196(.034)$ & $.176(.027)$ & $.097(.009)$ & $(3,33)$ & $61.69^{*}$ & .849 \\
\hline$\sigma$ & $16.018(1.800)$ & $19.500(4.281)$ & $23.868(6.686)$ & $37.528(7.153)$ & $(3,33)$ & $39.84^{*}$ & .784 \\
\hline
\end{tabular}

$\mathrm{YA}=$ younger adult; $\mathrm{OA}=$ older adult. Mean Weber contrast thresholds and slopes of the psychometric function (standard deviations in parentheses) in each of the four experimental conditions within the two age groups are shown in the first four columns. Also shown are the results of follow-up, withinsubjects ANOVAs performed on the log Weber contrast thresholds $(\mu)$ and the log slope parameter $(\sigma)$. ${ }^{a}$ Because Mauchly's test of sphericity was significant, $\chi^{2}(5)=14.29, p=.014(\hat{\varepsilon}=.755)$, the degrees of freedom for the $F$ statistics and significance levels reported are Greenhouse-Geissercorrected. ${ }^{*} p<.001$

greater difficulty than younger adults, overall, in segregating the target letter from the masker. This result is consistent with previous results - in both the visual and auditory domainsthat older adults' performance is more detrimentally influenced by the presence of maskers than is younger adults.

However, the significant interaction between age and masker type demonstrates that these age-related differences were not equal for different masker types. When the

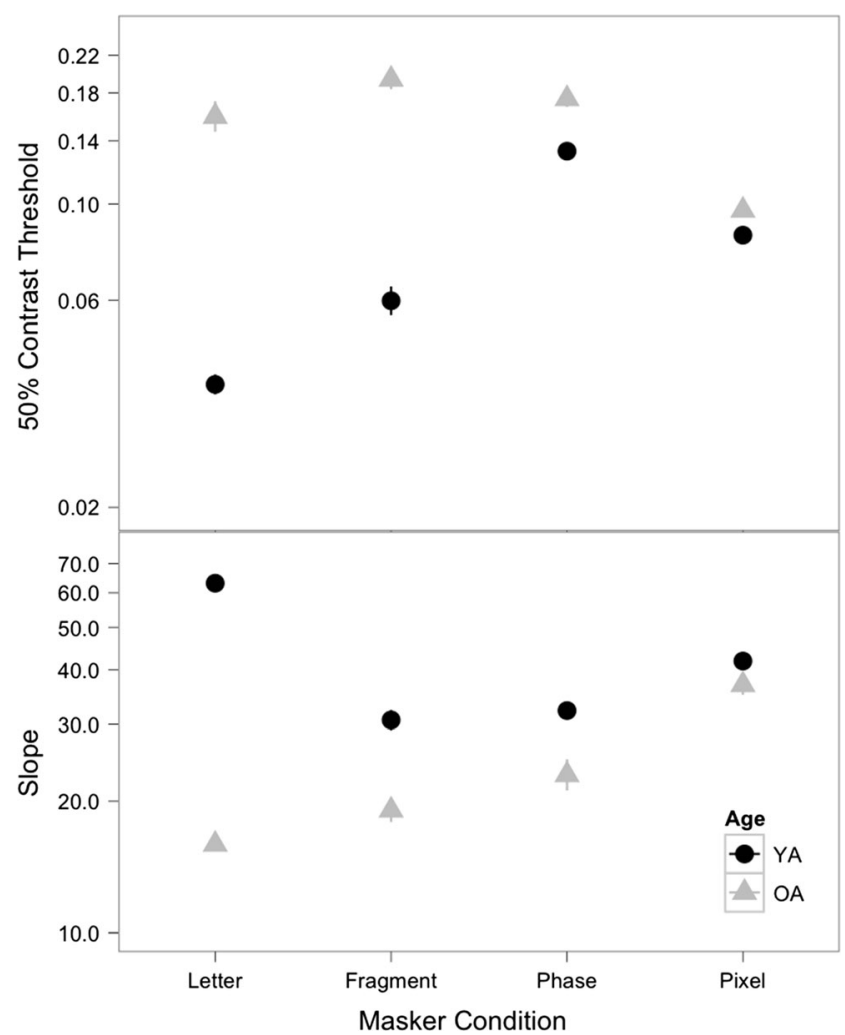

Fig. 4 Mean younger and older adult age group fitted $50 \%$ threshold and slope parameters by masker condition. Error bars represent $\pm 1 S E M$. Because the ANOVAs were conducted on the logarithms of the threshold and slope values, the spacing on the ordinate is logarithmic. $\mathrm{YA}=$ younger adult, $\mathrm{OA}=$ older adult concurrently presented mask was constructed (i.e., the pixel masker) by randomly locating the pixel luminances of a letterfilled masker, the magnitude of the difference between younger and older adults, although significant, was relatively small: Older adults, on average, required contrast levels that were just $14 \%$ darker than those of younger adults to achieve $50 \%$ correct levels of letter identification performance in the pixel masker condition. In contrast, presenting the to-beidentified letters in a surround composed of entire letters produced the largest age-related differences: In the letter masker condition, older adults required a level of contrast just over $320 \%$ darker than younger adults' to achieve equivalent letter identification performance. It is worth pointing out that even though our sample size for each age group was modest, these effects are so robust that the $95 \%$ confidence interval for the difference in thresholds between older and younger adults

Table 4 Pair-wise differences in log-transformed $50 \%$ thresholds between masker conditions in Experiment 1, for older and younger adult age groups separately

\begin{tabular}{lllll}
\hline & Letter & Fragment & Phase & Pixel \\
\hline YA & & & & \\
$\quad$ Letter & - & $-.192^{* *}$ & $-.537^{* * *}$ & $-.343^{* * *}$ \\
Fragment & & - & $-.345^{* * *}$ & $-.151^{* *}$ \\
Phase & & - & $.194^{* * *}$ \\
Pixel & & & & - \\
OA & & $-.085^{* *}$ & -.040 & $.216^{* *}$ \\
Letter & - & - & .045 & $.301^{* * *}$ \\
Fragment & & & - & $.256^{* * *}$ \\
Phase & & & & - \\
Pixel & & & & \\
\hline
\end{tabular}

$\mathrm{YA}=$ younger adult; $\mathrm{OA}=$ older adult. Differences are in the form row column, so positive values indicate that the column condition has a lower comparative $50 \%$ contrast threshold than the row condition. Significance levels are Bonferroni-corrected for the number of pair-wise comparisons within each age group. ${ }^{*} p<.05 .{ }^{* *} p<.01 .{ }^{* * *} p<.001$ 
Table 5 Pair-wise differences in log-transformed slopes between masker conditions in Experiment 1, for older and younger adult age groups separately

\begin{tabular}{|c|c|c|c|c|}
\hline & Letter & Fragment & Phase & Pixel \\
\hline \multicolumn{5}{|l|}{ YA } \\
\hline Letter & - & $0.314^{* * *}$ & $0.292^{* * *}$ & $0.179^{* * *}$ \\
\hline Fragment & & - & -0.021 & $-0.135^{* *}$ \\
\hline Phase & & & - & $-0.114^{\mathrm{a}}$ \\
\hline Pixel & & & & - \\
\hline \multicolumn{5}{|l|}{$\mathrm{OA}$} \\
\hline Letter & - & -0.179 & $-0.160^{*}$ & $-0.365^{* * *}$ \\
\hline Fragment & & - & -0.081 & $-0.287^{* * *}$ \\
\hline Phase & & & - & $-0.206^{* *}$ \\
\hline Pixel & & & & - \\
\hline
\end{tabular}

$\mathrm{YA}=$ younger adult; $\mathrm{OA}=$ older adult. Differences are of the form rowcolumn, so positive values indicate that the row condition has a steeper slope than the column condition. Significance levels are Bonferronicorrected for the number of pair-wise comparisons within each age group. ${ }^{*} p<.05 .{ }^{* *} p<.01$. $^{* * *} p<.001 .^{\mathrm{a}} p=.052$

ranges between percentage increases from $240 \%$ to $405 \%$. Hence, we can be very confident that the age difference in this condition was quite substantial.

What could account for older adults' massively worse performance than that of younger adults with the letter masker? On the surface, this result appears consistent with inhibitory deficit theories of aging, which predict that older adults should perform particularly poorly in conditions in which the distracting visual items are meaningful and could potentially interfere semantically with identification of the letter. However, the results of this experiment, as a whole, do not support an inhibition-based interpretation of the differences between age groups.

Contrary to our initial predictions, in the younger adult age group, the letter masker was associated with the lowest $50 \%$ contrast thresholds and the steepest psychometric function slopes, indicating that the presence of surrounding letters had the least impact on letter detection performance. For older adults, this relative benefit associated with the letter masker was not as evident. Two crucial related questions, therefore, emerge from the results of Experiment 1. The first is what type of process might allow younger adults to perform so well in a background of surrounding letters, relative to backgrounds composed either of letter fragments or of visual noise whose amplitude spectrum was identical to that in the letter masker. The second is how does aging virtually eliminate the processing advantage that younger adults possess when the target letter is surrounded by other letters?

With regard to the latter question, looking at the results of Experiment 1 as a whole provides some further clues to the nature of the age-related effect. When contours were eliminated from the letter masker by randomizing phase (creating the phase maskers, with visual noise with the same spatial frequency content) or by randomizing pixel location (creating the pixel masker, with visual noise with a uniform spectrum), age differences were relatively minimal. The addition of similar spatial frequency content in the phase masker relative to the pixel masker, further, had the similar effect of raising the $50 \%$ contrast thresholds in both age groups, indicating that younger and older adults alike were sensitive to the spatial frequency content of these two noise maskers. The patterns of performance for the younger and older adult age groups diverged markedly, however, when there were contours in the masker (the letter and fragment maskers). For younger adults, these two masking conditions were associated with significant, substantial reductions in contrast thresholds relative to the uncontoured noise in the phase and pixel maskers. Older adults, however, did not appear to gain this same benefit from the presence of contour information; $50 \%$ correct contrast thresholds were statistically indistinguishable in the fragment and phase masker conditions within the older adult age group. As a result, the gap between older and younger adults' performance widened substantially in the fragment masker condition as compared to that in the phase masker condition. Whereas older adults required target contrast levels just over $30 \%$ greater than younger adults with the uncontoured distractors in the phase masker condition, older adults required target letter contrast levels over $200 \%$ greater ( $95 \%$ confidence interval: lower bound $166 \%$, upper bound $294 \%$ ) than those required by younger adults with the contoured distractors of letter fragment condition. This suggests that the organization of the visual field into contoured objectsas occurs in the fragment maskers - is at least partially responsible for the even larger age-related effect found in the letter masker condition.

A second potential source of the age-related differences found in the letter masker condition is the familiar nature of the letters. In younger adults, there was an additional and substantial benefit from the formation of the contours into familiar letters (letter fragment vs. letter maskers). In contrast, older adults reaped a much smaller benefit from the familiarity of the letters. Moreover, older adults appeared to receive no benefit from the presence of contours alone (thresholds for the fragment masker condition did not differ significantly from those for the phase masker condition), but instead required those visual contours to form familiar objects to receive any benefit at all. It is worth noting that this pattern, as well, appears to contradict an inhibitory-deficit-type account of the age-related results in this experiment. If differences in the ability to inhibit meaningful and potentially interfering concurrent visual items could account for the age-related performance differences in this task, one would expect to find evidence that the semantically meaningful nature of the letter masker was contributing to the relative difficulty of letter detection in that masker condition. However, we here found the 
opposite: Older adults' performance was significantly better in the letter condition than with the visually similar - but unfamiliar and nonsemantic-masker composed of letter fragments.

Still, the benefit that older adults gained in the letter masker condition appeared to be smaller in magnitude than that in younger adults: Relative to the fragment masker condition, younger adults' thresholds were about $40 \%$ lower in the letter masker condition, whereas older adults' thresholds were only reduced by about $16 \%$. The overall pattern present in the slopes of the fitted psychometric functions reinforces this notion that younger adults reap a larger benefit from familiar letters than do older adults: Whereas younger adults' slopes in the fragment and phase masker conditions were similar, the letter masker was associated with a drastic steepening of the slopes, suggesting that some aspect of the letter masker allowed younger adults to rapidly and effectively segregate the distractors from the target letter. Because this steepening of slopes was only seen in the letter masker condition and not in the fragment masker condition, contour information alone does not appear to be enough to allow for this rapid segregation process. Older adults' slope data indicate that they were not benefiting from the organization of contours into letters, since the letter masker condition was not associated with steeper slopes, relative to the other masker conditions, in this age group.

Note, however, that the phase maskers used here had the same spatial frequency content as the letter maskers. However, as Fig. 2 shows, the spatial frequency content of the phase maskers, although similar to that of the fragment maskers, was not identical. To ensure that the larger-magnitude difference between the letter and phase maskers than between the fragment and phase maskers found here was not influenced by this factor, we performed a follow-up experiment that would allow a direct comparison between the letter fragment masker and its own phase-transformed masker condition.

An additional, independent sample of eight younger $(M=$ 19.0 years, $S D=.93)$ and eight older $(M=77.2$ years, $S D=$ 6.7) adults therefore completed the letter identification task under two masking conditions: with the original fragment maskers, and in a phase-fragment masker condition, which contained a new set of maskers that were phase transforms of the original fragment maskers, computed in the same way as the phase transforms of the letter maskers, described above. All other aspects of the experimental procedure, experimental setup, and psychometric fitting of the individual percentage correct letter identification data were the same as described above (see Table 1 for the vision screening measures for this group). The relative difference between the $50 \%$ contrast thresholds for the fragment masker and the phase masker in Experiment 1 (OA diff $=.05 \log$ units, YA diff $=-.35$ $\log$ units) closely resembled the relative difference between those for the fragment masker and its own phase- transformed maskers in this follow-up (OA diff $=.04 \mathrm{log}$ units, YA diff $=-.31 \log$ units), suggesting that the results discussed above were not due to differences in the spatial frequency content between the original phase maskers and the fragment maskers.

Two striking results, therefore, emerge from this experiment. The first is the effects of contoured information on younger adults' and older adults' performance. In masking conditions in which contours were present, versus visual noise having the same or similar spatial frequency content, younger adults appeared to be able to take advantage of this information, requiring lower signal strength to correctly identify the target letter. Older adults, in contrast, received little to no benefit from the presence of contour information in the masker, relative to noise maskers with the same or similar spatial frequency content. Second, both younger and older adults, remarkably, were better able to distinguish a letter among a field of similar, familiar letters than they were among a field of visually similar but unfamiliar letter fragments. However, the magnitude of this familiarity benefit appears to be influenced by age: The percentage reduction in thresholds associated with the letter versus the fragment masker in older adults was not even half as large as it was for younger adults.

A potential factor that could account for these age differences in performance is age-related slowing in visual processing. Older adults may simply need more time for the emergence of figure-ground relationships in the letter masker and letter fragment conditions, and/or for the recognition of these objects as familiar shapes. In order to assess the potential contribution of this factor, we conducted a similar experiment, varying the durations of the concurrently presented target letters and maskers.

\section{Experiment 2: speed}

One possible explanation for the results in Experiment 1 is that older adults simply need longer to process the incoming visual display, perhaps particularly so when higher-order processing might be required to effectively distinguish the letters from the masker (i.e., in the letter masker condition). This need for a longer stimulus processing time might be predicted by a generalized-slowing account (e.g., Salthouse, 1996); if agerelated processing speed declines lead to slower processing of incoming visual information, then older adults may need longer stimulus durations in order to achieve the same levels of performance as younger adults. If generalized slowing could account for the results seen in Experiment 1, we predicted that older adults should be more influenced by stimulus duration than are younger adults. Furthermore, longer stimulus durations than the $1 \mathrm{~s}$ used in the first experiment should improve performance for older adults particularly, and reduce 
the gap between older and younger adults' performance across all conditions.

To test the role of stimulus duration in the pattern of results seen in Experiment 1, separate groups of older and younger adults completed the same letter identification task under the same four masking conditions as in Experiment 1, but saw the letters and maskers at two different stimulus durations in each condition, one faster and one slower than the original duration (1 s) used. If increased processing time required by the older adults could account for the results found in Experiment 1, we expected to see a large influence of stimulus duration on older adults' performance in particular, with this effect being most pronounced in those conditions in which contours were present (i.e., the letter and fragment maskers).

\section{Method}

Participants Twelve younger adults ranging in age from 18 to 23 years old $(M=19.6, S D=1.4)$ and 12 older adults ranging from 67 to 79 years old $(M=74.5$ years, $S D=4.4)$ completed the study. As in Experiment 1, all participants were native speakers of English, with no history of neurological or ocular pathology, and completed the same in-lab vision screening described above, with near acuity levels of at least 20/25 (for the screening measure descriptives, see Table 1).

Materials and procedure Participants completed each of the four masker conditions described above, using the same masker stimuli and experimental setup. Each of the four masker conditions was presented at two possible stimulus durations: 250 and 1,750 ms. For each combination of stimulus interval and masker type, participants completed two blocks of 80 trials (20 trials at each of the four possible contrasts) wherein target letter contrast was randomly varied across trials. Participants received a short (approximately 1.5-min) break between blocks. The two blocks for each of the two stimulus durations within each masker condition were blocked together, and all blocks for each masker condition were blocked together. The order of the two possible stimulus durations within each masker condition block was counterbalanced within participants, such that each participant saw each stimulus duration first or second an equal number of times (twice). The order of the two blocks at each stimulus duration within each condition block and the relative order of the condition blocks were also counterbalanced across the participants in each age group, such that each of the two possible stimulus duration orders was presented an equal number of times within each condition, and the relative order of each of the condition blocks was presented an equal number of times. Older and younger adults completed the same set of counterbalances so that each group as a whole saw the same set of possible block orders. Prior to the two consecutive experimental blocks at each stimulus duration for each condition, participants completed eight practice trials of that stimulus duration, two at each of the four possible contrasts, presented in a random order.

\section{Results}

Figure 5 displays the average proportion correct letter identification performance as a function of target letter contrast, at each stimulus duration (indicated by shape and color) for the four masker conditions (panel rows) and the two age groups (panel columns). These average performance data are displayed along with fitted psychometric functions, of the same form as is described above in Experiment 1.

Overall, these data show a clear effect of stimulus duration, such that the curves fit to the average data for letters and maskers presented concurrently for $250 \mathrm{~ms}$ (black curves and circles) are shifted to the right along the $x$-axis as compared to the curves fit to the average data for masker-plusstimulus durations of $1,750 \mathrm{~ms}$ (gray curves and triangles), indicating that the participants in both age groups tended to require higher levels of contrast for target letter identification

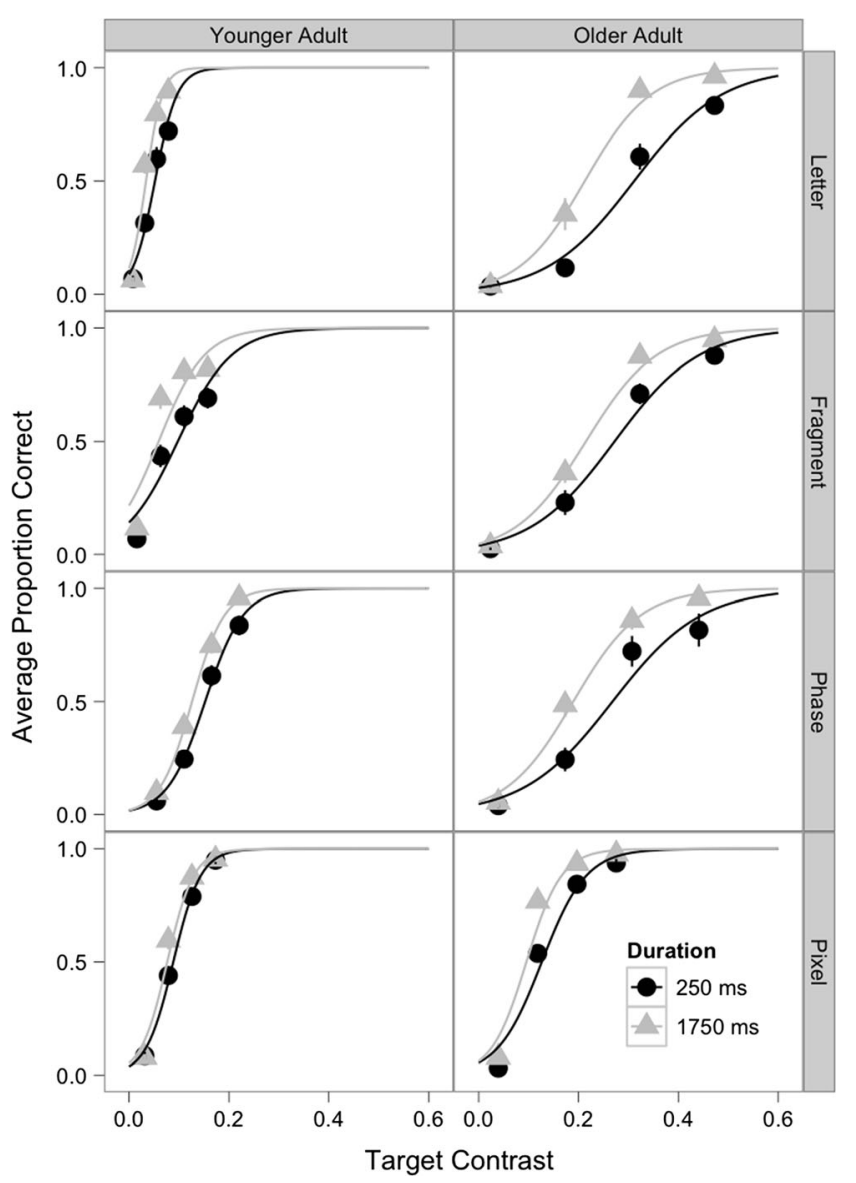

Fig. 5 Average percent correct target letter identification as a function of contrast for younger and older adult age groups in the four masker conditions, with psychometric functions fitted to the participant-average data in Experiment 2. Error bars represent \pm 1 SEM 
when the stimulus duration was shorter. However, the spacing between the curves within each panel varies substantially, indicating differences in the effects of stimulus duration for the different masker conditions. In particular, for both older and younger adults, the two separate curves are relatively close together in the pixel condition, indicating that stimulus duration had relatively little effect when the masking noise was composed of randomly organized pixels with spatial frequency content unrelated to that of the target letters. For the masking conditions in which the spatial frequency content of the maskers was similar to that of the letter targets (i.e., the letter, fragment, and phase conditions), stimulus duration appears to have more of an effect, shifting curves for shorter durations further to the right than the longer-duration curves.

Comparing older and younger adults, an examination of the longer-duration functions (gray curves) suggests that - even with increased stimulus durations relative to Experiment $1-$ older adults' psychometric functions remain substantially shifted to the right and shallower than the younger adults' functions at both stimulus durations in the phase, fragment, and letter conditions. This pattern indicates that increasing the presentation time did relatively little to close the performance gap between older and younger adults seen in Experiment 1, particularly for those masking conditions in which the frequency content of the maskers was similar to that of the target letters. An examination of the effect of stimulus duration on the psychometric functions within each age group does suggest that duration may have had a larger effect on the older than the younger adults. However, since older adults' curves at both stimulus durations are still shifted substantially to the right - into a range of higher contrast levels - relative to the younger adults' curves, the same absolute difference in the threshold for older adults may in fact represent smaller percent changes in threshold than among the younger adults.

To confirm whether these patterns seen in the average data characterized the individual data, the log-transformed individual psychometric function thresholds and slopes were each analyzed using a $2 \times 2 \times 4$ mixed-design ANOVA with Age Group (young, old) as a between-subjects factor and Masker Condition (letter, pixel, phase, fragment) and stimulus duration $(250,1,750 \mathrm{~ms})$ as within-subjects factors. ${ }^{3}$ For younger adults, in the fragment masker condition with stimuli presented for $1,750 \mathrm{~ms}$, five out of 12 participants had lower percent correct levels for the darkest contrast level than for the second-

\footnotetext{
${ }^{3}$ Two younger adults (one in the fragment and letter conditions at $250 \mathrm{~ms}$ and one in the phase condition at $250 \mathrm{~ms}$ ) and one older adult (in the phase condition at $250 \mathrm{~ms}$ ) failed to achieve greater than $50 \%$ correct performance at the darkest contrast for a masker condition. In addition, the data from the phase masker condition at $1,750 \mathrm{~ms}$ for one older adult were not available due to experimenter error. Data from these conditions for these participants are thus not included in the analyses described.
}

to-darkest contrast level. As a result, the traditional psychometric function provided poor fits and deflated the estimated $50 \%$ contrast thresholds for this condition. To ensure consistent comparisons in the ANOVA for this study, the individual $50 \%$ contrast thresholds for each condition and stimulus duration were therefore estimated using a linear interpolation around the two contrast levels that bracketed $50 \%$ performance for each participant. Slopes, similarly, were estimated around the $50 \%$ threshold point using percentage performance at the two contrast levels bracketing $50 \%$ performance.

Converging with the results of Experiment 1, the $2 \times 2 \times 4$ ANOVA on the individual estimated $50 \%$ thresholds demonstrated significant main effects of both condition and age group (see Table 6 for all main effects and interactions), indicating that, overall, thresholds varied significantly between the different masking conditions and also between the different age groups. Also replicating the effects of Experiment 1, we observed a significant two-way interaction between age and condition, indicating that (collapsing across stimulus durations) the effect of age on the thresholds differed across conditions.

Consistent with the predictions outlined above, stimulus duration had a significant main effect on the contrast thresholds; the two-way interaction between condition and stimulus duration was also significant, confirming that, collapsing across age groups, the effects of stimulus duration differed across the four masking conditions. However, in contrast to the hypotheses outlined above, the two-way interaction between age and stimulus duration only approached significance $(p=.165)$. The three-way interaction between age group, masker condition, and stimulus duration was significant,

Table 6 Main ANOVA results performed on the log-transformed $50 \%$ thresholds $(\mu)$ and log-transformed slope values $(\sigma)$ for Experiment 2

\begin{tabular}{lllll}
\hline & $d f$ & $F$ & $\eta_{\mathrm{p}}{ }^{2}$ & $p$ \\
\hline$\mu$ & & & & \\
$\quad$ Age & $(1,18)$ & 222.79 & .925 & $<.001$ \\
Masker Type & $(3,54)$ & 97.10 & .844 & $<.001$ \\
Duration & $(1,18)$ & 198.38 & .917 & $<.001$ \\
Age $\times$ Masker Type & $(3,54)$ & 197.55 & .916 & $<.001$ \\
Age $\times$ Duration & $(1,18)$ & 2.10 & .104 & .165 \\
Masker Type $\times$ Duration & $(3,54)$ & 6.78 & .274 & .001 \\
Age $\times$ Masker Type $\times$ Duration & $(3,54)$ & 5.81 & .244 & .002 \\
& & & & \\
$\quad$ Age & $(1,18)$ & 192.12 & .914 & $<.001$ \\
Masker Type & $(3,54)$ & 32.71 & .645 & $<.001$ \\
Duration & $(1,18)$ & 44.76 & .713 & $<.001$ \\
Age $\times$ Masker Type & $(3,54)$ & 51.83 & .742 & $<.001$ \\
Age $\times$ Duration & $(1,18)$ & 1.916 & .096 & .183 \\
Masker Type $\times$ Duration & $(3,54)$ & 4.89 & .214 & .004 \\
Age $\times$ Masker Type $\times$ Duration & $(3,54)$ & 2.82 & .136 & .047 \\
\hline
\end{tabular}


suggesting the presence of between-condition differences in the comparative effects of stimulus duration across the two age groups.

As Fig. 5 suggests, both older and younger adults received a benefit - a lowering of threshold (release from masking) from increasing stimulus durations by $1.5 \mathrm{~s}$. To explore the nature of the three-way interaction noted above, we therefore computed the magnitude of this duration-associated release from masking for each individual by subtracting - for each masking condition - the log of the $50 \%$ contrast thresholds for the 1,750-ms stimulus durations from the log-transformed thresholds for the 250-ms stimulus durations. Between-agegroup pair-wise comparisons (for which the reported $p$ values are Bonferroni-corrected for multiple comparisons) in each masker condition indicated that older and younger adults' duration-associated release from masking did not differ significantly in the letter, $t(21)=1.83, p=.32$, phase, $t(19)=$ $2.20, p=.16$, or pixel, $t(22)=1.74, p=.384$, conditions, but younger adults had a significantly greater release from masking in the fragment condition, $t(21)=3.12, p=.02$ (see Fig. 6).

The lack of a robust age difference in the effects of stimulus duration is reinforced by a parallel analysis on psychometric function slopes (Table 6), not discussed in detail here. Younger adults' psychometric function slopes showed a significant stimulus duration by condition interaction, $F(3,27)=4.52, p$ $=.011, \eta_{\mathrm{p}}{ }^{2}=.334$; this interaction emerged from a lack of significant effect of stimulus duration on psychometric function slopes for the uncontoured phase and pixel masker conditions ( $p \mathrm{~s}>.25)$, but a significant effect of stimulus duration on the slopes in the letter and fragment conditions $(p \mathrm{~s}<.01)$, with the largest magnitude difference being in the fragment condition (Fig. 6). For older adults, in contrast, a marginally significant stimulus duration by condition interaction $[F(3$, 27) $\left.=2.75, p=.062, \eta_{\mathrm{p}}{ }^{2}=.234\right]$ reflected fewer betweencondition differences in the effects of stimulus duration on the psychometric function slopes (Fig. 6).

\section{Discussion}

The results of this study clearly demonstrate that stimulus duration can have an influence on participants' ability to identify a letter amidst visual distractors. For both age groups and across all conditions, shorter stimulus durations were associated with an increase in the signal strength required to successfully identify a letter. However, the magnitude of the influence of stimulus duration was not consistent across different types of visual distractors, and the relative impacts of stimulus duration across these different types of visual distractors were not consistent between older and younger adults.

The results of Experiment 2 are inconsistent with the hypothesis that the patterns of behavior observed in Experiment
1 could be explained by hypothesizing that older adults required more time to process visual information than did younger adults. If older adults simply needed more time to perform the letter identification task than did younger adults, then we would expect larger improvements in performance as a function of stimulus duration for older than for younger adults, particularly in those conditions in which the age-related differences were the largest (letter maskers and fragment maskers). However, when improvement in performance was measured on a log scale, older adults, on average, did not benefit more than younger adults from increases in stimulus duration. Moreover, the largest-magnitude improvements in performance due to an increase in stimulus duration were observed in younger adults in the letter and fragment masker conditions (see Fig. 6). In addition, a comparison of younger and older adults' $50 \%$ identification thresholds for stimulus durations of 1,000 ms (Exp. 1) to those at durations of 1, $750 \mathrm{~ms}$ (Exp. 2) indicated that increasing the stimulus duration by $750 \mathrm{~ms}$ for the most part had very little influence on the thresholds, with the average benefit from a $750-\mathrm{ms}$ increase in duration ranging from .12 log units (letter masker condition) to $.02 \log$ units (pixel masker condition) for younger adults, and from -.10 log units (letter masker condition) to $.05 \mathrm{log}$ units (phase masker condition) for older adults.

Interestingly, even with $250 \mathrm{~ms}$ exposure to the stimulus and maskers, younger adults were able to accurately identify letters at much lower signal strengths when they were copresented with other letters than when they were copresented with either letter fragments or the two kinds of visual noise. Hence, the processes that permit younger adults to benefit from the perceptual or cognitive nature of the letter shapes are available at relatively short exposure durations, roughly equivalent to a "glance." This reinforces the notion that the time it takes to process the stimulus displays, at least with respect to the time scales employed here, cannot explain the pattern of age-related differences found here.

However, the results of Experiment 2 do reinforce the importance that visual contours may play in the masker type and age effects revealed in Experiment 1 when the maskers are devoid of semantic content (letter fragment maskers, random phase maskers, and random pixel maskers). For younger adults, increased stimulus durations were associated with the largest reduction in log contrast thresholds and the greatest steepening of psychometric function slopes when the target letter was viewed concurrently with the letter fragment masker. For older adults in the letter fragment masker condition, the effect of stimulus duration was comparatively weak, and not larger than the effect of stimulus duration for the two types of visual noise maskers for either thresholds or slopes. It thus appears that younger adults reap a much larger benefit from increased display times when there are contours in the semantically meaningless visual distractors (letter fragment maskers vs. random phase or random pixel maskers) than do older 


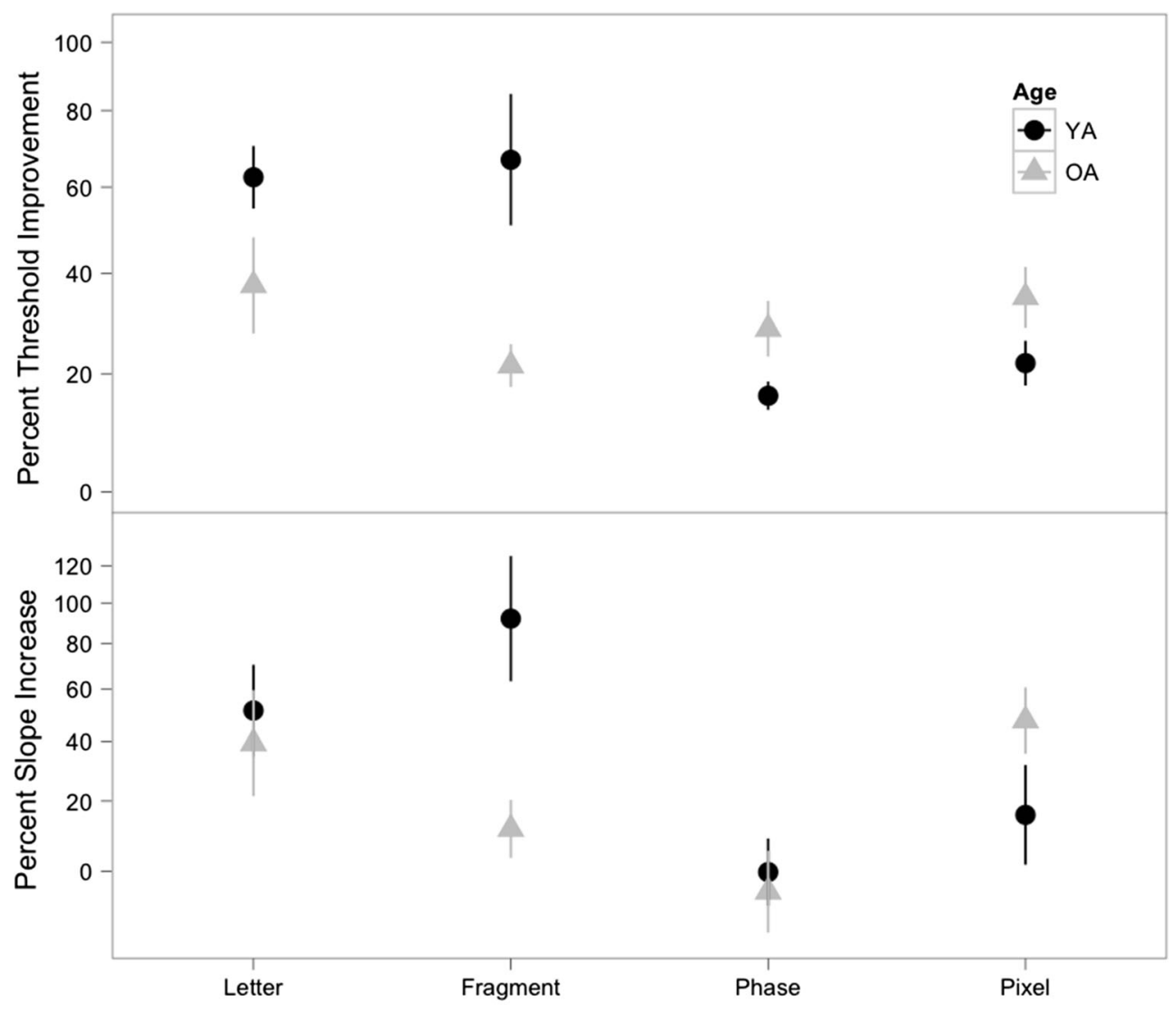

Masker Condition

Fig. 6 Percentage improvement in thresholds and steepening of slopes associated with an increase in stimulus duration from 250 to $1,750 \mathrm{~ms}$. The percentage improvement in thresholds was calculated as

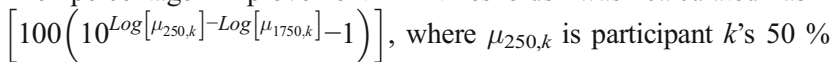
threshold when the stimulus duration was $250 \mathrm{~ms}$ and $\mu_{1750, k}$ is participant $k$ 's $50 \%$ threshold when the stimulus duration was $1,750 \mathrm{~ms}$. The percentage steepening of the slope was calculated as

adults. Moreover, in both Experiments 1 and 2, younger adults benefited more than older adults from the presence of contours in semantically meaningless visual distractors. These results suggest that older adults do not benefit as readily as do younger adults from the presence of contour information in a visual masker.

One possible explanation for the failure of older adults to profit from the availability of contours in a semantically meaningless visual masker is that their reduced contrast sensitivity may result in less robust contours. Indeed, with poorer and poorer contrast sensitivity, the boundaries of the letter and letter fragment object contours would become increasingly less sharp, and the appearance of the maskers would approach the visual qualities of the phase masker. This would explain why, when the masker was devoid of semantic content, the performance of older adults in the presence of letter fragment maskers was equivalent to their performance in visual noise having the same spatial frequency content (random phase maskers). Moreover, if the boundaries of the individual $\left[100\left(10^{\log }\left[\sigma_{1750, k}\right]-\log \left[\sigma_{250, k}\right]-1\right)\right]$, where $\sigma_{250, k}$ is participant $k$ 's $50 \%$ threshold when the stimulus duration was $250 \mathrm{~ms}$ and $\sigma_{1750, k}$ is participant $k$ 's $50 \%$ threshold when the stimulus duration was $1,750 \mathrm{~ms}$. Note that the percentage improvement in threshold and the percentage steepening of the slope associated with an increase in duration are plotted in logarithmic coordinates. Error bars represent \pm 1 SEM. YA = younger adult, $\mathrm{OA}=$ older adult

masking objects were less sharp, this might impose bottlenecks on processing such that the familiarity of the letters would not be as easily detected, or would not unfold in a time course that was able to support early-level object segregation. In order to investigate the influence of reduced contrast sensitivity on performance under these visual masking conditions, we performed a study similar to Experiment 2 in a separate younger adult sample who viewed maskers and letter stimuli filtered to replicate the decreased contrast sensitivity of older adults (Exp. 3).

\section{Experiment 3: contrast filter}

To assess the part that older adults' decreased contrast sensitivity might play in the pattern of results seen in Experiments 1 and 2, we sought to replicate the contrast sensitivity losses experienced by older adults in a young adult sample. A separate group of younger adults completed the same letter 
identification task under the same four masking conditions as in Experiments 1 and 2, but they saw versions of the letters and maskers that were filtered to decrease the image contrast in a way comparable to the spatial-frequency-specific contrast sensitivity losses associated with aging. If age-related contrast sensitivity losses could account for the large age-related differences seen in Experiment 1, we expected that younger adults viewing the targets and maskers under these filtered conditions would no longer differ significantly from the older adult age group who performed the first experiment.

\section{Method}

Participants Eight younger adults ranging in age from 19 to 21 years $(M=19.8$ years, $S D=0.71)$ completed the study. As in Experiments 1 and 2, all participants were native speakers of English with no history of neurological or ocular pathology, and completed the same in-lab vision screening. To ensure comparability in visual function between the younger adults in this study and the older adult age groups from the previous experiments, the younger adults were recruited with near acuity levels that sampled from the range of near acuity levels present in the set of older participants in Experiments 1 and 2. Given the percentage differences between the older and younger adults across all conditions in Experiment 1 and our previous power analysis (see the Appendix), we settled on eight younger adults for this sample, since this sample size would allow us to (a) maintain our counterbalanced order of conditions, (b) sample from the entire acuity range of older adults in the first study, and (c) provide sufficient power to detect each of our main experimental effects of interest in Experiment 1. The resulting eight individual younger adults in this experiment still generally tended to perform better on the contrast sensitivity screening, but they had exactly matched measured near acuity, and nearly exactly matched far acuity (see Table 1 for all vision screening descriptives), as compared to the individual older participants in the eight-participant comparison subsets of older adults included in Experiment $1\left(M_{\mathrm{age}}=\right.$ $\left.68.75, S D_{\text {age }}=4.03\right)$ and Experiment $2\left(M_{\text {age }}=74.25, S D_{\text {age }}\right.$ $=4.80)$.

Materials and procedure Participants completed each of the four masker conditions described in Experiment 1, using the same base masker stimuli and experimental setup. Each of the four masker conditions was presented at two possible stimulus durations: the original $1 \mathrm{~s}$ from Experiment 1, and $250 \mathrm{~ms}$. Prior to the presentation of each target letter and masker, maskers and targets were filtered with a custom filter to lower the image contrast in a way comparable to what would be experienced by older adults, based on sample-estimated (Owsley, Sekuler, \& Siemsen, 1983) average contrast sensitivity for a 70-year-old. To simulate in young adults the image contrast experienced by the older adults in these experiments, the experimental stimuli were first Fourier-transformed. Second, the spatial frequency of each of the sinusoidal gratings in this Fourier decomposition was determined [spatial frequency in cycles $/$ degree $=\sqrt{\left(\epsilon^{2}+\eta^{2}\right)}$ ]. Third, each of these sinusoidal gratings was filtered according to the profile depicted in Fig. 7, which was derived from the spatial-frequency - specific differences in log contrast sensitivity between 20-year-olds and 70-year-olds reported in Owsley et al. (1983, Table 5). Also shown in Fig. 7 are the losses in contrast sensitivity (solid data points) experienced by the 70-year-old participants in Owsley et al. (1983), vis-à-vis the 20-year-old participants in that study. As Fig. 7 shows, the amplitude profile of the filter used here to simulate age-related loss of contrast sensitivity provides a reasonable fit to the data from Owsley et al. (1983). Note, however, that the filter employed here assumed that the age-related loss of contrast sensitivity as a function of spatial frequency is independent of the orientation of the gratings in the Fourier decomposition. All other aspects of the experimental setup and procedure were identical to those described in Experiment 2.

\section{Results}

Figure 8 plots the average proportions of correctly identified target letters at each contrast level in the each of the conditions (panel rows), at each of the two stimulus durations (panel columns), separately for younger (black circles) and older (gray triangles) adults, along with the fitted smooth psychometric functions of the form described in Experiment 1. For younger adults, the data plotted are the results of Experiment 3 , in which younger adults viewed contrast-filtered versions of the maskers and the to-be-identified target letters. For older adults, the data plotted represent the average data from the subset of acuity-matched older adults from Experiment 1 (1,

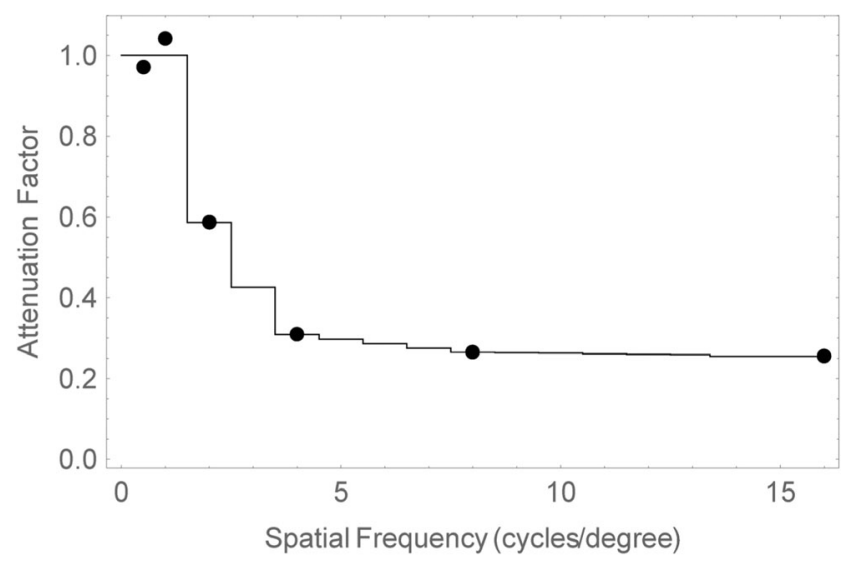

Fig. 7 Attenuation factor (line) applied to the experimental stimuli in Experiment 3, based on the age-related differences in contrast sensitivity at each spatial frequency (plotted circles) reported in Owsley et al. (1983) 


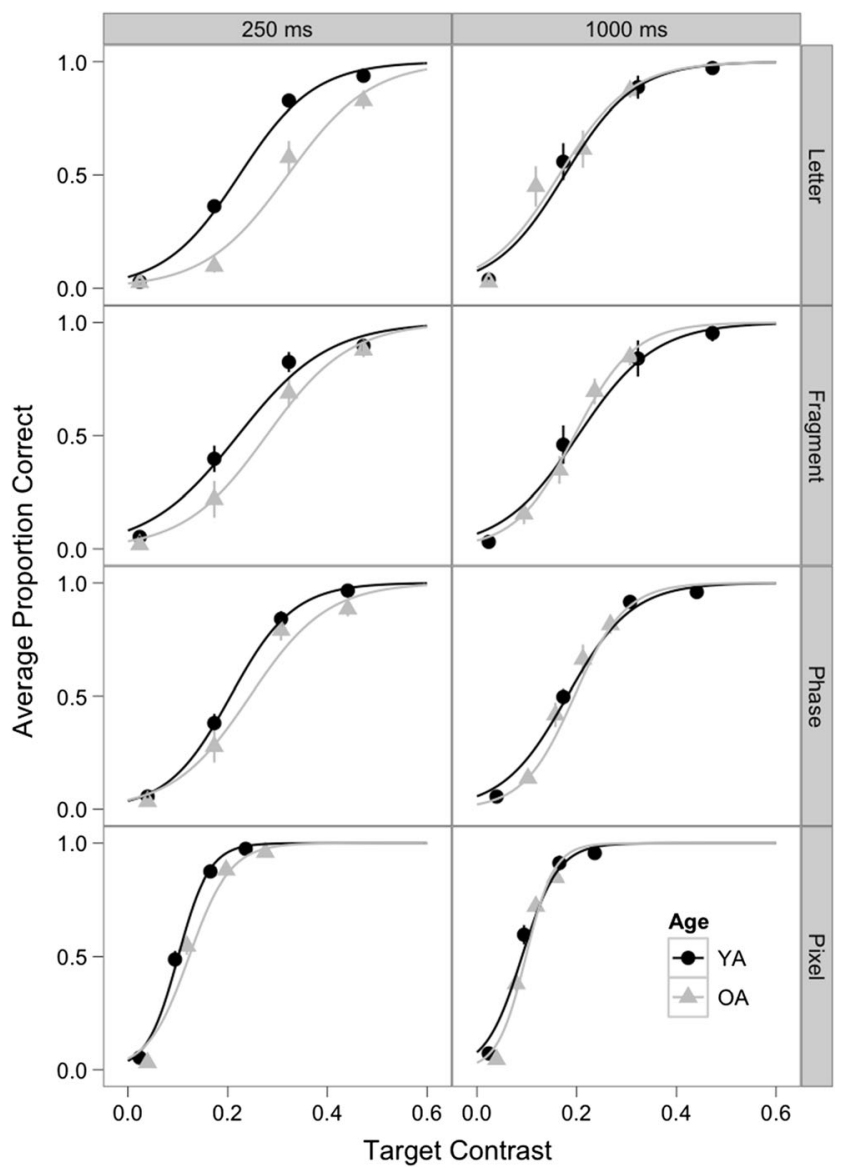

Fig. 8 Average percent correct target-letter identification as a function of contrast for younger adults viewing contrast-filtered targets and stimuli in Experiment 3, and for the acuity-matched older adults from Experiments 1 and 2, at the two stimulus durations in the four masker conditions, with psychometric functions fitted to the participant-average data. Error bars represent $\pm 1 S E M . \mathrm{YA}=$ younger adult, $\mathrm{OA}=$ older adult

000-ms average performance and fitted functions) and Experiment 2 (250-ms average performance and fitted functions).

An examination of the second column $(1,000 \mathrm{~ms})$ of Fig. 8 clearly shows that when the contrast information available in the filtered stimulus displays presented to younger adults corresponded to the contrast information available to older adults in the unfiltered stimulus displays (in which contrast information was reduced due to age-related losses in contrast sensitivity), the performance of younger adults was virtually equivalent to that of older adults. Hence, the large age-related differences that characterized performance in the letter and letter fragment conditions in Experiment 1 (see Fig. 3) were eliminated.

To confirm whether these average patterns characterized the individual data, psychometric functions were fitted to the individual data in each condition, and the $\log$ of the fitted slope and threshold parameters were retained for further analysis, as described above (for summary statistics for the individual fitted functions by age and masker condition, see Table 7). To investigate the impact of filtering the stimuli presented to younger adults to match the contrast sensitivity of the average 70-year-old, the data from these young adults were analyzed along with the data from acuity-matched older adults in a mixed-design ANOVA with Age Group (young filtered vs. old, unfiltered, acuity-matched) as a betweensubjects factor and Masker Condition (letter, fragment, phase, pixel) as a within-subjects factor. For the log thresholds, we found a main effect of masker condition, $F(3,42)=63.49, p<$ $.001, \eta_{\mathrm{p}}{ }^{2}=.819$, but no main effect of age group, $F<1, p=$ .972 , or age by masker type interaction, $F<1, p=.698$, confirming that filtering the stimuli presented to younger adults to match the contrast sensitivity of older adults eliminated the differences in log thresholds found between older and younger adults in Experiment 1. Similarly, a parallel analysis using a mixed-design ANOVA on the individual psychometric function slopes, $\sigma$, for the 1-s stimulus duration also revealed a main effect of masker condition, $F(3,42)=16.49, p$ $<.001, \eta_{\mathrm{p}}{ }^{2}=.541$, but no main effect of age group, $F<1, p=$ .694 , or age by masker type interaction, $F=1.65, p=.193$.

The results are somewhat different, however, at the shorter stimulus duration examined in Experiment 2 (Fig. 8, left column). When stimulus durations were shorter, the gap between the older adults in Experiment 2 and the younger adults in Experiment 3 who were presented the filtered stimulus displays began to reappear, with the older adult functions shifted again relatively rightward along the $x$-axis. At shorter stimulus durations, the older adults from Experiment 2 still required higher contrast levels to achieve performance equivalent to that of younger adults viewing contrast-filtered maskers and stimuli in the present experiment. Although younger adults' performance appears relatively unchanged across the two stimulus duration conditions of Experiment 3 (Fig. 8, black functions, comparing across the right and left columns), older adults appear to be more negatively impacted by shortened stimulus durations (Fig. 8, gray functions, comparing across the right and left columns). This suggests that contrast sensitivity differences between older and younger adults cannot account for the differing effects of stimulus duration on performance for younger and older adults found in Experiment 2, and cannot fully account for age-related differences when the viewing times are short.

A 2 (age group: young with filtered stimuli vs. old with unfiltered stimuli) $\times 4$ (masker condition) between-within ANOVA on the logarithms of the individual psychometric function slopes for the $250-\mathrm{ms}$ condition, as above, revealed a significant effect of masker condition, $F(1.76,24.66)=$ $38.35, p<.001, \eta_{\mathrm{p}}{ }^{2}=.733$, but no main effect of age group, $F<1, p=.998$, or age by masker type interaction, $F<1, p=$ .515 (note that the degrees of freedom and significance values are Greenhouse-Geisser-corrected where appropriate). A parallel $2 \times 4$ between-within ANOVA on the individual psychometric function thresholds for the 250-ms condition alone, however, revealed significant main effects of both masker 
Table 7 Means and standard deviations for thresholds and slopes for the younger adults in Experiment 3 and acuity-matched subsamples of older adults from Experiments 1 and 2

\begin{tabular}{|c|c|c|c|c|c|c|c|c|}
\hline & \multicolumn{2}{|l|}{ Letter } & \multicolumn{2}{|l|}{ Fragment } & \multicolumn{2}{|l|}{ Phase } & \multicolumn{2}{|l|}{ Pixel } \\
\hline & 250 & 1,000 & 250 & 1,000 & 250 & 1,000 & 250 & 1,000 \\
\hline \multicolumn{9}{|l|}{ YA } \\
\hline$\mu$ & $.223(.026)$ & $.179(.062)$ & $.221(.044)$ & $.207(.084)$ & $.206(.030)$ & $.182(.029)$ & $.100(.014)$ & $.091(.021)$ \\
\hline$\sigma$ & $13.775(2.850)$ & 23.105 (16.980) & $11.798(2.906)$ & $18.813(7.591)$ & $17.098(3.458)$ & $18.787(6.627)$ & 30.907 (3.087) & $31.118(9.495)$ \\
\hline \multicolumn{9}{|l|}{$\mathrm{OA}$} \\
\hline$\mu$ & $.321(.059)$ & $.166(.059)$ & $.276(.071)$ & $.196(.042)$ & $.244(.051)$ & $.185(.030)$ & $.122(.038)$ & $.098(.011)$ \\
\hline$\sigma$ & $13.118(2.785)$ & $16.030(2.050)$ & $13.582(2.486)$ & $18.157(3.981)$ & $20.297(18.66)$ & $21.571(5.961)$ & $29.625(8.590)$ & $37.368(8.001)$ \\
\hline
\end{tabular}

$\mathrm{YA}=$ younger adult; $\mathrm{OA}=$ older adult. Mean Weber contrast thresholds and slopes of the psychometric function (standard deviations in parentheses) in the four conditions are shown for two durations of stimulus presentation ( 250 and 1,000 ms) for both age groups. For older adults, the threshold and slope values for were taken from a subset of eight individuals from Experiment 2 (250-ms stimulus duration) and a subset of eight individuals from Experiment 1 (1,000-ms stimulus duration). Both subsets were matched in acuity to the eight young individuals in Experiment 3 . For all cells, $N=8$

condition, $F(1.81,25.27)=144.63, p<.001, \eta_{\mathrm{p}}{ }^{2}=.912$, and age group $F(1,14)=9.24, p<.01, \eta_{\mathrm{p}}{ }^{2}=.397$, indicating that the contrast filter did not eliminate age-related differences in threshold for these shorter stimulus durations. The age by masker type interaction was not significant with this sample size, and the effect size was relatively small, $F(1.81,25.27)=$ $1.61, p=.221 \eta_{\mathrm{p}}{ }^{2}=.103$ (note, as above, that that the degrees of freedom and significance values are Greenhouse-Geissercorrected where appropriate).

\section{Discussion}

The results of this study indicate that, for 1,000-ms presentation times, when younger adults viewed stimuli that "looked" like what older adults would see, their performance closely mirrored that of the older adults in Experiment 1. Younger adults who performed the same letter identification task from Experiment 1 with contrast-filtered stimuli exhibited average psychometric functions and slopes that mirrored an acuitymatched sample of older adults in every masker condition. Task performance with these contrast-filtered stimuli eliminated any significant differences between younger adults and a the subsample of older adults from Experiment 1, who were matched in acuity.

However, the performance still remained significantly different between younger adults viewing these contrast-filtered stimuli for $250 \mathrm{~ms}$ and an acuity-matched subsample of older adults from Experiment 2 who also viewed the stimuli for $250 \mathrm{~ms}$, with older adults requiring elevated contrast thresholds in every condition, as compared to younger adults. This suggests that older adults do experience, for relatively fast stimulus durations, speed-related restrictions to performance that are not accounted for by declines in visual acuity or contrast sensitivity. However, the magnitude of these betweenage-group differences attributable to speed remained relatively small as compared to the initial results of Experiment 1, which appear to be well accounted for by contrast sensitivity declines alone.

\section{General discussion}

At the outset of this article, we wondered whether the apparent support for age-related inhibitory deficits found in previous work in the visual domain might, instead, be accounted for by lower-level perceptual changes that occur with advancing age. To investigate this question, we used a letter identification task, in which participants attempted to identify a letter presented concurrently with different types of visual distractors. This task allowed us to investigate access to meaningful, semantic stimuli in the face of visual distractors, with an experimental paradigm free from the potential confounds inherent to typically used reading-with-distraction paradigms. By varying the signal strength of the to-be-identified letter relative to simultaneously presented visual maskers and fitting psychometric functions to the resulting accuracy data, we were able to derive estimates of both the relative difficulty (psychometric function $50 \%$ accuracy thresholds) and efficiency (psychometric function slopes) of task performance in the face of different types of distracting visual information.

For both older and younger adults, we expected that the presentation of a visual masker composed of other letters would be particularly detrimental to letter identification performance, due to the visual similarity between the target and masker, and to the presence of potentially interfering semantic content in the masking visual items (i.e., nontarget letters). As a result, we expected that psychometric function thresholds would be inflated in the letter masker condition relative to other masking conditions. Inhibitory deficit accounts also predict that the detrimental effect of meaningful semantic content in the maskers should be more severe in older than in younger adults. Hence, age-related differences in $50 \%$ accuracy 
thresholds, under an inhibitory deficit account, should be greatest when the elements of the masker are semantically meaningful and potentially interfering (i.e., the letter masker condition), in comparison to visually similar but meaningless distractors (i.e., the fragment and phase masker conditions).

In Experiment 1, we found that the presence of semantically meaningful distractors did, indeed, produce the largest agerelated differences in letter identification performance: The magnitudes of the differences in psychometric function slopes and thresholds between older and younger age groups were far greater in the letter masker condition than in any other masker condition. In the letter masker condition, younger adults required greatly reduced signal intensity, in the form of target letter contrast, to reach levels of letter detection accuracy commensurate to those of older adults; younger adults' psychometric functions also exhibited much steeper slopes, suggesting that they experienced less task interference from the presence of the masker. However, this age-related difference emerged in an unexpected way.

Because we examined letter detection accuracy in the face of several different visual "controls" for the distracting maskers, we were able to observe that the large age-related difference in the letter masker condition did not appear because older adults were more detrimentally affected than younger adults by the addition of semantic content to the visual masker. Rather, large age-related differences emerged because younger adults were much better at letter detection when the concurrently presented masker was composed of letters than in any other masker condition, whereas older adults were not. Younger adults had vastly reduced psychometric function thresholds in the letter masker condition relative to the other masking conditions; older adults, in contrast, showed relatively similar threshold levels across the letter, fragment, and phase masker conditions, with reductions in thresholds (much smaller in magnitude than those for younger adults) only emerging in the letter condition.

This pattern of results is quite different than what one would expect under an inhibitory deficit account. If either age group were having trouble inhibiting the letters in the masker, one would expect that the addition of familiarity and semantic meaningfulness would create more difficulty in the letter masker condition than in the fragment masker condition - not less, as we found here. What, then, might account for the age-related differences found in the first experiment?

The results of Experiment 1 as a whole provide some clues as to the nature of these age-related differences in letter detection performance amidst visual distractions. For both age groups, when the spatial frequency content of the noise masker was similar to that of the target letters (phase masker), $50 \%$ correct contrast thresholds were greater than when the noise masker had the same RMS value but with a uniform spatial frequency content (pixel masker). However, only younger adults benefited when those same frequencies present in the phase masker were organized into contoured but lexically meaningless objects (the fragment masker); the thresholds of older adults for the fragment masker did not improve significantly when compared to the phase masker. This pattern of results hints that, for younger adults, the organization of the spatial frequency content in the visual noise into contours improves the degree of segregation of the target object from the rest of the masking field. If older adults were less able to form these contours, the pattern of results described above would be predicted.

In Experiment 2, we tested whether age-related reductions in processing speed may have contributed to older adults' apparent inability to use the contour information in Experiment 1 . If older adults needed longer to form visual contours than the stimulus duration employed in Experiment 1 allowed, they might not have had enough time to reap the benefits that fully formed contours provided to younger adults in the fragment and letter masker conditions. Although older adults did appear to be somewhat more influenced by stimulus duration overall than younger adults, the increased stimulus durations in Experiment 2 did essentially nothing to close the gaps in performance between older and younger adults found in Experiment 1. Indeed, the largest effects of stimulus duration in any condition were in fact found in the younger adult age group, for whom increased stimulus durations brought $50 \%$ contrast thresholds for the fragment masker condition closer to those found in the letter masker condition. This result supports the idea that object segregation via contour information unfolds over time, but the results, considered as a whole, suggest that the time limitations of this process cannot provide an adequate account of the results in Experiment 1. Older adults, on the other hand, showed essentially the same effect of stimulus duration in the fragment and phase conditions, as would be expected if contour information were completely unavailable to object segregation processes.

In Experiment 3, we tested the possibility that age-related reductions in visual function might impair contrast sensitivity to such a degree that contour formation is disrupted, leading to impeded task performance. Younger adults performed the same letter identification task as in Experiments 1 and 2, but with filtered versions of the stimuli that approximated the spatial-frequency-dependent contrast sensitivity typically experienced by an older adult. The results of Experiment 3 revealed that decreasing contrast sensitivity in this way was enough to replicate, within a younger adult age group, the levels and patterns of relative difficulty in each type of masking noise found in the older adult age group in Experiment 1 when the stimuli were displayed for $1,000 \mathrm{~ms}$. These results support the idea that the age-related effects seen in the first experiment largely depended on contrast sensitivity losses with increasing age. Without appealing to age-related differences in higher-order processing, the performance patterns of older adults were replicable in a younger adult age 
group - simply by decreasing their contrast sensitivity to the incoming visual stimulus in a way that mimicked losses seen in normal aging. However, when the stimulus duration was shortened to $250 \mathrm{~ms}$, the younger adults presented with filtered stimuli still outperformed older, acuity-matched adults, indicating that, even after controlling for age-related differences in contrast sensitivity, visual processing takes longer for older than for younger adults.

An open question remains of how exactly the type of visual information present in the letter masker was able to improve performance relative to other visually similar controls, at least when contour formation was intact. For both age groups, the addition of contours that made up actual letters (in the letter masker condition) resulted in further reductions in thresholds over the fragment condition's nonletter formed contours. This result, along with the steepness of the psychometric function curves for the letter masker condition in Experiment 1, indicates that some quality of the letter masker allowed younger adults to be extremely efficient in segregating the target letter from the masker. Since the other masker types included transforms of the letter maskers that retained their contour information (fragment masker), spatial-frequency information (phase masker), and overall contrast levels (pixel masker), it is unlikely that the information allowing rapid segregation of the letter masker from the target was purely perceptual in nature.

Rather, it appears that some other aspect of the visual content in the letter masker allowed younger adults, under normal viewing circumstances, to efficiently segregate the masking information from the target letter. One interpretation consistent with these results is that a visual benefit is afforded by the familiar nature of the letters (i.e., they are objects that have been seen a great deal before and can be tied to a long-term memory representation) in the letter masker, versus the unfamiliar fragments (which did not form any complete familiar objects) in the fragment masker. A number of previous results have suggested that a range of visual tasks are influenced when incoming visual information can be tied to extant long-term memory representations. Visual search is faster and less influenced by the field size of to-be-searched items if the items are familiar (i.e., letters in one's own alphabet) rather than unfamiliar (Malinowski \& Hubner, 2001; Wang, Cavanagh, \& Green, 1994); objects presented in the context of semantically related items are identified more accurately than when they are presented with unrelated objects (Auckland, Cave, \& Donnelly, 2007); semantic consistency in the relationship between objects and background scenes (i.e., a football player, rather than the pope, on a football field) facilitates identification of both the object and the scene (Davenport \& Potter, 2004).

One possible locus for the difference between the letter and fragment masker conditions, in younger adults, is thus that differences in the familiarity of the masker in the two conditions led to differences in the allocation of attentional resources. Humans are motivated to characterize novel or unfamiliar stimuli (e.g., the "orienting response"; Pavlov, 1927/2010; Sokolov, 1963), and the fragment, phase, and pixel masker conditions all contained visual elements that did not form readily recognizable shapes. If the visual novelty of the masking information in these masker conditions drew greater attentional resources than the letter shapes in the letter masker condition, then less attention may have been available for target letter identification in the nonletter masker conditions. When spatial-frequency resolution is poor, as in older age groups or the younger adults in Experiment 3, the correspondence of the incoming visual stimulus to a stored long-term memory representation may be reduced; this may have created-even in the letter masker condition - a distribution of attentional resources more closely resembling the profile in the nonfamiliar masking conditions, with corresponding elevation of thresholds and greater interference from the masker.

Another possibility is that the familiar nature of the components in the letter maskers facilitated object formation, assisting with target-masker segregation. Although a variety of results have shown that object recognition can be aided by the familiarity/consistency of either the object itself or the surrounding visual context, why these effects obtain has remained controversial, with disagreement over whether familiarity assists later processes of object recognition by allowing, for example, for more informed "guesses" as to object identity once a basic perceptual representation of the object has been formed (e.g., Hollingworth, 1998), or whether familiarity can, instead, assist during the initial, objectforming stages of perception (e.g., Davenport, 2007; Davenport \& Potter, 2004). Recently, Kahan and Enns (2014) showed that (familiar) letters were less susceptible than unfamiliar, letter-like stimuli to a type of masking that interferes with boundary formation and edge assembly. These results are consistent with a role for familiarity in the very early stages of object processing, in which boundaries and edges are assigned to individual objects in the field of view. Extending these results to the present task, if the object edges belonging to familiar objects are more easily recognized and categorized, then in the letter masker condition - in which both the target letter and masking visual information were familiar (letters) the segregation of the masking objects from the target letter may have been especially robust. Indeed, the drastically steeper psychometric function slopes associated with the letter masker condition in Experiment 1 is consistent with this interpretation, as it suggests that younger adults in this condition were more able to effectively segregate the masker from the target than when the distracting background consisted of a set of unorganized contours (fragment masker) or of visual noise. The effect of manipulating contrast sensitivity in Experiment 3 could be seen as consistent with this interpretation, as well; to the extent that the loss of contrast sensitivity for high- 
spatial-frequency information results in fuzzier object boundaries, if the beneficial effect of familiarity was working on object boundary formation, the effect of familiarity should be lessened when contrast sensitivity for higher spatial frequencies is lessened. Future work will be needed to determine the extent to which these results are consistent with differences in attentional deployment, or a role for familiarity in very early stages of object formation and figure-ground segregation, or some combination of the two.

Whatever the precise nature of this familiarity benefit, the results of these experiments do not support the need to appeal to age-related losses in the ability to apply long-term memory representations to the incoming visual stimulus to explain the differences seen between older and younger adults. Older adults showed significantly lower thresholds in the letter masker than in the fragment masker condition in Experiment 1, and also displayed larger effects of stimulus duration in the letter masker condition in Experiment 2 than in any other. These results suggest that, even though the familiarity information itself may be less accessible, the ability to apply this top-down knowledge to the visual task at hand may be largely intact. Indeed, the fact that the older adult age group did display significantly lower $50 \%$ identification thresholds in the letter masker condition than in the fragment masker condition indicates that they, too, can benefit when a set of contours in the masker can be organized into a set of familiar and semantically meaningful objects. The present results for older adults also indicate that when a set of contours cannot be so organized, the effect on performance is no different than that of visual noise having the same spatial frequency content.

These results thus highlight the profound impact that sensory changes can have on cognitive life with increasing age. Taken together with the results of Experiment 1, the results of Experiment 3 suggest that, with normal aging, older adults may experience contrast sensitivity losses of sufficient magnitude that contour formation is devastatingly disrupted. Our manipulation that restricted younger adults' contrast sensitivity, in Experiment 3, was enough to eliminate the apparent massive benefit of contours, reflected in the lower contrast thresholds for the letter and fragment masker conditions, relative to the phase masker, in the young adult age group in Experiment 1 . Crucially, this loss of contrast sensitivity was also apparently enough to eliminate the very large benefit of familiar letters seen in the young adult age group in Experiment 1: The younger adults in Experiment 3 had $50 \%$ contrast thresholds indistinguishable from those of the older adult age group in Experiment 1, and the magnitude of the familiarity benefit of the letter masker over the fragment masker was much more similar between the older adults in Experiment 1 ( $22 \%$ reduction in threshold for the letter masker) and the contrast-impeded younger adults in Experiment 3 (14 \% reduction in threshold) than between the older adults in Experiment 1 and the younger adults in Experiment 1 (50\% reduction in threshold). This effect of reducing high-spatialfrequency contrast information suggests that intact, robust contour formation may be necessary to take advantage of object familiarity.

Consistent with accounts of age-related cognitive changes that stress the role of lower-order sensory changes (Burke \& Shafto, 2004), the results of these experiments thus emphasize the potential role that relatively lower-order sensory issues can play in apparently higher-order task performance. In addition, Faubert (2002) has argued that the deleterious effects that agerelated deficits in lower-order visual processes (such as contrast sensitivity) have on the performance of visually dependent tasks can become progressively more severe, as the degree to which these tasks engage larger and larger neural networks becomes greater. Clearly, extracting and recognizing a target letter from a background of other letters is likely to engage wider and more complex neural networks than those required to identify letters in visual noise. We would, accordingly, expect the loss of contrast sensitivity to have more severe consequences when the masker consists of letters than when it is composed of visual noise.

Hence, reduced contrast sensitivity and associated impaired contour formation may set older adults up for a host of issues in performing a variety of types of higher-order visual tasks. Because an overlapping set of sensory factors likely limits performance in both our letter identification task and the reading-with-distraction paradigm previously used in the majority of work supporting age-related inhibitory deficits, the apparent higher-order, age-related declines previously found in reading-with-distraction performance may well also emerge from age-related sensory changes similar to those explored in the present set of experiments. Recall that in a typical readingwith-distraction task, participants must read and comprehend "target" text, while ignoring interspersed "nontarget" text that is distinguished from the target text by some difference in lower-order visual features (most often italics; see, e.g., Mund et al., 2010). At a basic level, the results from the experiments presented here strongly suggest that older and younger adults vastly differ in their abilities to perceive crucial, contourrelated visual features of individual letters. Age-related declines in contrast sensitivity and contour formation therefore likely would have - as they do in the letter identification task in this article's set of experiments - a profound impact on the ability to perceive differences between fonts. Given the crucial role that font discrimination (i.e., italics from nonitalics) plays in the typical reading-with-distraction task paradigm, if older adults are less able to visually discriminate targets from distractors, their task performance should be substantially worse than that of their younger adult counterparts. Of note, this interpretation aligns with prior experimental findings showing that altering the visual characteristics of the distracting text can change the magnitude of age-related differences in reading-with-distraction task performance. When 
distinguishing distractor text from target text relies on the perception of a visual feature that is likely heavily influenced by contour formation (e.g., letter slant; Connelly, Hasher, \& Zacks, 1991; Mund et al., 2010), age-related differences obtain; when distractors differ from the target text via visual features not likely to rely heavily on contour formation (e.g., color: Kemper, McDowd, \& Kramer, 2006; Phillips \& Lesperance, 2003; or spatial location: Carlson et al., 1995), age-related differences are drastically reduced or eliminated.

Future work using reading-with-distraction paradigms and the appropriate visual controls for the intervening information will be needed to outline which of these factors are at play, and how fully a sensory-based account of the type we have developed here can explain the variety of results typically found in these types of tasks. One particularly interesting approach may be to degrade visual stimuli in a way consistent with our method in Experiment 3 and to compare the effects of such sensory degradation in younger adults to the pattern of performance typically seen in older adults' reading-withdistraction performance. Comparing the changes to performance and types of errors (i.e., slower reading, more interference from semantically related words) exhibited by younger adults under such degraded visual conditions could be particularly informative as to the downstream results of age-related declines in visual function.

Since object formation is so central to everyday visual tasks, our results may also have implications for additional higher-order, heavily visually demanding tasks in which older adults are particularly prone to difficulty-for example, driving a motor vehicle (Owsley, 1999). Timely response to events during driving requires rapid identification of potentially ambiguous objects in the environment: a deer that may potentially run in front of a car, or a pedestrian not paying attention while crossing the street. If object identification in these scenarios, in a young visual system, normally receives crucial support from contextual factors (schema or normal patterns of co-occurrence) that older adults are not as able to take advantage of, this lack of contextual benefit could contribute to performance deficits (i.e., slowed reaction times due to slowed object recognition). In other words, the complex visual task of driving may not be particularly difficult for older adults because they are more distracted by events unfolding along the course of the road, or because they require a longer time for the processes of object recognition, but because younger adults' robust incoming sensory representations allow them to particularly quickly and effortlessly segregate figure and ground and identify everyday objects that can be linked to long-term memory representations.

In sum, the results of the present set of experiments highlight the crucial role that sensory factors may have in a variety of complex, higher-order tasks that rely on visual input. These experiments support the notion that many age-related issues in such tasks may emerge not because older adults have a deficit in higher-order processing, per se, but because lower-level sensory changes prohibit older adults from taking advantage of cues (e.g., object familiarity or supporting contextual information) that greatly assist task performance in a young, healthy, and normally functioning visual system. Under this account, lower-level visual changes can thus, in effect, set older adults up to fail at higher-order tasks, not because the actual processes required for task performance are impaired, but because the input to those tasks is impoverished in such a way that it interferes with the normal course of processing. The results presented here thus highlight the importance of future work detailing the role that losses in contrast sensitivity and benefits from familiar object properties and/or visual schemas may play in age-related changes in perceptual and cognitive performance. To the extent that more peripheral processes (i.e., optical changes, etc.) may be easier to improve than more central processes (i.e., changes in processing speed or inhibitory abilities), this may be a somewhat hopeful result and suggests potential avenues for investigation that may result in improvements to real-world age-related declines in functioning.

Author Note This research was supported by grants from the Canadian Institutes of Health Research (Grant No. MOP-15359, TEA-12497) and Natural Sciences and Engineering Research Council of Canada (Grant No. RGPIN-9952-13). The authors thank Michelle Okewa, Auni Aushun, Mohammed Altantawi, and Michelle Bae for their assistance in the data collection.

\section{Appendix}

The power to detect a theoretical difference between the means of two groups of participants depends on three parameters: the size of the difference to be detected between the means of the two groups, the variability of the within-group measures, and the sample size $n$. The between-group differences that we were primarily interested in were the young-old threshold differences for the letter condition and the letter fragment condition. The other two conditions, random phase and random pixel, were within-subjects control conditions designed to control for spectral composition and RMS contrast.

In order to determine our sample sizes for Experiment 1, we used the following procedure to estimate the power associated with a hypothesized mean difference between two age groups. First, we selected the range of contrast values to be employed in each of the eight conditions ( 2 Ages $\times 4$ Conditions) on the basis of pilot experiments (a common practice in psychophysics). Specifically, we varied the range of contrasts employed to define a range in each condition that we could reasonably expect to bracket the $50 \%$ threshold. We adjusted each range so that the midpoint of the range was our best guess as to the contrast corresponding to the average participant's 
$50 \%$ threshold, and spaced the middle two contrast values at a distance from each other such that it was likely that an individual's $50 \%$ threshold would fall within these two values. When we made these adjustments on the basis of pilot data, it became obvious that the range increased across conditions as the expected value of the $50 \%$ threshold increased. When we used the range as a proxy for the standard deviation of the measures and plotted the range as a function of the expected mean value for a condition, we noted that the range was roughly proportional to the mean $(r=.8)$. Hence, we expected the standard deviation of the thresholds to be roughly proportional to the mean. It is known that when the standard deviation is proportional to the mean, a log transformation will produce data such that the standard deviations of the logtransformed data will be roughly the same across conditions.

Finally, we made the assumption that $80 \%$ of the log threshold values in a condition would fall between the logarithms of the second and third contrast values employed in a condition, with the central value of the distribution being the logarithm of the center of the range. Under these assumptions, it follows that

$-1.28155=\frac{\log \left[C_{2}\right]-\log \left[C_{\mathrm{M}}\right]}{\sigma}$,

where $C_{2}$ is the second lowest contrast of the four contrasts in a condition, and $C_{\mathrm{M}}$ is the contrast associated with the midrange. A plot of $\sigma$ as a function of $\log \left[C_{\mathrm{M}}\right]$ showed that the standard deviation was roughly constant across the eight conditions. The mean standard deviation across the eight conditions was 0.073 . Hence, for the log-transformed threshold values, we assumed that the standard deviation of the values in each of the conditions was .073 .

Given this estimate of the standard deviation, we were then able to determine, for a given number of participants in each group, the power to detect a specified difference in the mean $\log$ values between younger and older adults. The mean log differences in thresholds that yielded a power equal to .8 for $n$ $=4,8,12$, and 16 participants in each group were .147056 , $.09597188, .0767527$, and $.0658405 \log$ units, respectively. These values correspond to percentage increases in threshold for older adults over younger adults of $40 \%, 25 \%, 19 \%$, and $16 \%$, respectively, for sample sizes of $4,8,12$, and 16 participants per age group. A multiple of four participants per age group would allow us to maintain the counterbalance of masker condition within each age group. We therefore opted for 12 participants per group because of diminishing improvements as the sample size was successively increased by four participants. Hence, when $n=12$ and the standard deviation of the $\log$ thresholds in a condition was .073 , the probability of detecting a $19 \%$ increase in threshold, $\{100-[100$ (older adults' thresholds)/(younger adults' thresholds)]\}, would be .8 in all conditions.
Of course, these probability calculations depend on the assumption that the standard deviation of the log threshold values in each condition was .073 . We checked how reasonable this assumption was by examining the obtained standard deviations of the log threshold values in the eight conditions of Experiment 1. When we examined the standard deviations of the $\log 50 \%$ thresholds in each condition, we found that they ranged from .031 to .121 , with a mean value of .072 . Hence, assuming a standard deviation of the log threshold values of .073 in each condition was reasonably close to the actual mean log threshold values across conditions. Accordingly, for Experiment 2, we simply maintained our original 12-person sample size.

For Experiment 3, we wanted to recruit a sample of younger adults who were entirely acuity-matched to the sample of older adults in Experiment 1. We therefore chose a reasonable sample size of younger adults that would allow us to detect age-related differences commensurate to, or even substantially reduced from, the age-related effects found in Experiment 1. Using the logic above, using eight participants would allow us to detect a significant effect if the thresholds for older adults were $25 \%$ larger than those of the younger adults, a percentage increase that was less than $1 / 8$ the size of the age-related effect found in Experiment 1 for our two main conditions of interest.

\section{References}

Auckland, M. E., Cave, K. R., \& Donnelly, N. (2007). Nontarget objects can influence perceptual processes during object recognition. Psychonomic Bulletin \& Review, 14, 332-337. doi:10.3758/ BF03194073

Brebion, G. (2001). Language processing, slowing, and speed/accuracy trade-off in the elderly. Experimental Aging Research, 27, 137-150. doi:10.1080/036107301750073999

Brebion, G. (2003). Working memory, language comprehension, and aging: Four experiments to understand the deficit. Experimental Aging Research, 29, 269-301. doi:10.1080/03610730303725

Burke, D. M., \& Shafto, M. A. (2004). Aging and language production. Current Directions in Psychological Science, 13, 21-24. doi:10. 1111/j.0963-7214.2004.01301006.x

Carlson, M. C., Hasher, L., Connelly, S. L., \& Zacks, R. T. (1995). Aging, distraction, and the benefits of predictable location. Psychology and Aging, 10, 427-436. doi:10.1037/0882-7974.10.3.427

Chung, S. T. L., \& Tjan, B. S. (2009). Spatial-frequency and contrast properties of reading in central and peripheral vision. Journal of Vision, 9(9), 16. doi:10.1167/9.9.16

Connelly, S. L., Hasher, L., \& Zacks, R. T. (1991). Age and reading: The impact of distraction. Psychology and Aging, 6, 533-541. doi:10. 1037/0882-7974.6.4.533

Davenport, J. L. (2007). Consistency effects between objects in scenes. Memory \& Cognition, 35, 393-401. doi:10.3758/BF03193280

Davenport, J. L., \& Potter, M. C. (2004). Scene consistency in object and background perception. Psychological Science, 15, 559-564. doi: 10.1111/j.0956-7976.2004.00719.x

Duchek, J. M., Balota, D. A., \& Thessing, V. C. (1998). Inhibition of visual and conceptual information during reading in healthy aging 
and Alzheimer's disease. Aging, Neuropsychology, and Cognition, 5, 169-181. doi:10.1076/anec.5.3.169.616

Faubert, J. (2002). Visual perception and aging. Canadian Journal of Experimental Psychology, 56, 164-176. doi:10.1037/h0087394

Hasher, L., \& Zacks, R. T. (1988). Working memory, comprehension, and aging: A review and a new view. In G. H. Bower (Ed.), The psychology of learning and motivation: Advances in research and theory (Vol. 22, pp. 193-225). New York, NY: Academic Press. doi:10.1016/S0079-7421(08)60041-9

Hollingworth, A. (1998). Does consistent scene context facilitate object perception? Journal of Experimental Psychology: General, 127, 398-415. doi:10.1037/0096-3445.127.4.398

Kahan, T. A., \& Enns, J. T. (2014). Long-term memory representations influence perception before edges are assigned to objects. Journal of Experimental Psychology: General, 143, 566-574. doi:10.1037/ a0033723

Kemper, S., McDowd, J., \& Kramer, A. (2006). Eye movements of young and older adults while reading with distraction. Psychology and Aging, 21, 32-39. doi:10.1037/0882-7974.21.1.32

Lustig, C., Hasher, L., \& Zacks, R. T. (2007). Inhibitory deficit theory: Recent developments in a "new view.". In D. S. Gorfein \& C. M. MacLeod (Eds.), Inhibition in cognition (pp. 145-162). Washington, DC: American Psychological Association. doi:10. 1037/11587-008

Malinowski, P., \& Hubner, R. (2001). The effect of familiarity on visualsearch performance: Evidence for learned basic features. Perception \& Psychophysics, 63, 458-463. doi:10.3758/BF03194412

Mund, I., Bell, R., \& Buchner, A. (2010). Age differences in reading with distraction: Sensory or inhibitory deficits? Psychology and Aging, 25, 886-897. doi:10.1037/a0019508

Mund, I., Bell, R., \& Buchner, A. (2012). Aging and interference in story recall. Experimental Aging Research, 38, 20-41. doi:10.1080/ 0361073X.2012.636724

Owsley, C. (1999). Driver capabilities. Paper presented at the Transportation in an Aging Society: A Decade of Experience conference, Bethesda, Maryland.

Owsley, C. (2011). Aging and vision. Vision Research, 51, 1610-1622. doi:10.1016/j.visres.2010.10.020
Owsley, C., Sekuler, R., \& Siemsen, D. (1983). Contrast sensitivity throughout adulthood. Vision Research, 23, 689-699. doi:10.1016/ 0042-6989(83)90210-9

Pavlov, I. P. (2010). Conditioned reflexes: An investigation of the physiological activity of the cerebral cortex. Annals of Neurosciences, 17(3), (Original work published 1927). doi:10.5214/ans.0972-7531. 1017309

Pelli, D. G., \& Tillman, K. A. (2007). Parts, wholes, and context in reading: A triple dissociation. PLoS ONE, 2, e680. doi:10.1371/ journal.pone. 0000680

Phillips, N. A., \& Lesperance, D. (2003). Breaking the waves: Age differences in electrical brain activity when reading text with distractors. Psychology and Aging, 18, 126-139. doi:10.1037/ 0882-7974.18.1.126

Pichora-Fuller, M. K. (1997). Langauge comprehension in older listeners. Canadian Journal of Speech-Language Pathology and Audiology, 21, 125-142.

Salthouse, T. A. (1996). The processing-speed theory of adult age differences in cognition. Psychological Review, 103, 403-428. doi:10. 1037/0033-295X.103.3.403

Schefrin, B. E., Tregear, S. J., Harvey, L. O., Jr., \& Werner, J. S. (1999). Senescent changes in scotopic contrast sensitivity. Vision Research, 39, 3728-3736. doi:10.1016/S0042-6989(99)00072-3

Schneider, B. A., \& Pichora-Fuller, M. K. (2000). Implications of perceptual deterioration for cognitive aging research. In F. I. M. Craik \& T. A. Salthouse (Eds.), The handbook of aging and cognition (2nd ed., pp. 155-219). Mahwah, NJ: Erlbaum.

Sokolov, Y. (1963). Perception and the conditioned reflex. Oxford, UK: Pergamon Press.

Steenbekkers, L. (1998). Visual contrast sensitivity. In L. Steenbekkers \& M. Beijsterveldt (Eds.), Design-relevant characteristics of ageing users. Backgrounds and guidelines for product innovation (pp. 131-136). Delft, The Netherlands: Delft University Press.

Treisman, A. M., \& Gelade, G. (1980). A feature-integration theory of attention. Cognitive Psychology, 12, 97-136. doi:10.1016/00100285(80)90005-5

Wang, Q., Cavanagh, P., \& Green, M. (1994). Familiarity and pop-out in visual search. Perception \& Psychophysics, 56, 495-500. doi:10. 3758/BF03206946 